\title{
A computational model integrating brain electrophysiology and metabolism highlights the key role of extracellular potassium and oxygen
}

\author{
D Calvetti $^{1} \quad$ G Capo Rangel $^{2} \quad$ L Gerardo Giorda $^{2} \quad$ E Somersalo $^{1,2}$ \\ ${ }^{1}$ Department of Mathematics, Applied Mathematics and Statistics \\ Case Western Reserve University \\ ${ }^{2}$ Basque Center for Applied Mathematics
}

\begin{abstract}
The human brain is a small organ which uses a disproportional amount of the total metabolic energy production in the body. While it is well understood that the most significant energy sink is the maintenance of the neuronal membrane potential during the brain signaling activity, the role of astrocytes in the energy balance continues to be the topic of a lot of research. A key function of astrocytes, besides clearing glutamate from the synaptic clefts, is the potassium clearing after neuronal activation. Extracellular potassium plays a significant role in triggering neuronal firing, and elevated concentration of potassium may lead to abnormal firing pattern, e.g., seizures, thus emphasizing the importance of the glial $\mathrm{K}^{+}$buffering role. The predictive mathematical model proposed in this paper elucidates the role of glial potassium clearing in brain energy metabolism, integrating a detailed model of the ion dynamics which regulates neuronal firing with a three compartment metabolic model. Because of the very different characteristic time scales of electrophysiology and metabolism, care must be taken when coupling the two models to ensure that the predictions, e.g., neuronal firing frequencies and the oxygenglucose index (OGI) of the brain during activation and rest, are in agreement with empirical observations. The temporal multi-scale nature of the problem requires the design of new computational tools to ensure a stable and accurate numerical treatment of the problem. The model predictions for different protocols, including combinations of elevated activation and ischemic episodes, are in good agreement with experimental observations reported in the literature.
\end{abstract}

\section{Introduction}

Our understanding of how the human brain functions relies on measurements through different windows capturing diverse aspects of this multifaceted organ: The electrophysiological brain is monitored by using electroencephalography (EEG) or magneto-encephalography (MEG), metabolic functions are observed, e.g., using positron emission tomography (PET), whereas functional MRI, and in particular, the blood oxygenation dependent signal (BOLD) provide information about the cerebral blood flow. It is not uncommon for one modality to be used as a proxy for another, e.g., BOLD fMRI is used in cognitive studies to infer on the location of firing neurons, however this implicitly assumes that the hemodynamic and electrophysiological functions are spatially and temporally tightly coupled. The extent and mechanism of the coupling continue to be the topics of a lot of research using both experimental protocols and mathematical models.

Modeling any one cerebral aspect is a challenging task by itself; combining the models describing different processes in order to better understand the coupling is harder than the sum of challenges, in part because the wide difference in the time scales of the processes makes this a multi-scale models requiring specially designed computational tools. Because the characteristic time constants of electric activity, metabolic processes and blood flow in brain differ by orders of magnitude, from milliseconds to several minutes, the system of differential equations governing the integrated model is characterized by extreme stiffness. In addition to the problems rising from the wide range of 
time scales, many spatial details of the cerebral electro-metabolic units and the effect of diffusion are forgone in the spatially lumped models traditionally used to describe brain metabolism and hemodynamics.

In the present article we propose a computational framework that tracks the integrated effects of the electrophysiological and metabolic behavior of a neuron-astrocyte complex as it changes from rest to neuronal activation and it experiences an episode of transient ischemia. Computed experiments show that the predictions of the model are in agreements with experimentally findings reported in the literature.

The range of electrophysiology models based on the classical Hodgkin-Huxley formalism that can be found in the literature range from simple phenomenological models of neuronal spiking as the two-variable model in [29] to more complex, conductance-based models that consider ion fluxes between the intra- and extracellular space. Among the latter, some consider only the temporal evolution of the membrane potential [44], while others include the dynamics of ionic concentrations as well $[17,7,43,47]$. Our model of the electrophysiology is a modification and an extension of [17] which, in addition to the extracellular potassium, whose effect on neuronal firing is well understood, takes also into account the intracellular sodium dynamics, the $\mathrm{Na}^{+}-\mathrm{K}^{+}$pump activity and the potassium cleaning function of astrocytes. In particular, in this paper we seeks to shed some light on the metabolic consequences of the latter.

The computational models of brain metabolism proposed in the literature vary widely in detail and complexity; see [41] for an overview. It is quite popular to describe cellular brain metabolism by means of a spatially lumped well mixed compartment model governed by a system of ordinary differential equations which track the concentrations of relevant substances, see, e.g., [22, 5, 2, 3, 4, 16, 19]. It is well known that while the number of parameters in dynamic models of metabolic processes increases rapidly as details are added, a reduced model may not be able to capture the complexity of the dynamic behavior of the cells. Therefore to strike a balance between computational complexity and model resolution, our implementation of a two-way feedback mechanism between the electrophysiology and brain energy metabolism is based on the reduced four compartment metabolic model discussed in [41] comprising blood, extra-cellular space, neuron, and astrocyte compartments.

In our model the coupling of electrophysiology and metabolism occurs through the energy exchange: the electrophysiology is regulated through the availability of ATP produced by the metabolic processes and, conversely, the metabolism produces energy in response to the energetic need of the electrophysiology. The idea of coupling electrophysiology and brain metabolism is not new. Some of the metabolic models in the literature include intracellular sodium and potassium concentrations as state variables, and in [30], the ion fluxes are coupled with a Hodgkin-Huxley model. Our model elaborates further on the details of the two-way feedback mechanism between electrophysiology and metabolism, in particular emphasizing the energetic cost of the $\mathrm{K}^{+}$glial buffering. In fact, while the model in [30] includes an energetic cost for the $\mathrm{Na}^{+}-\mathrm{K}^{+}$pump coupled with the Hodgkin-Huxley model, it does not include the astrocyte cleaning of extracellular potassium. Stemming from the model proposed in [17], we introduce a dependence on the phosphorylation states of neuron and astrocyte by modifying both the $\mathrm{Na}^{+}-\mathrm{K}^{+}$pump and the $\mathrm{K}^{+}$glial buffering, thus ensuring a double feedback with the metabolism.

Because the electrophysiological changes happen in the millisecond range while the metabolic processes are in the scale of seconds or minutes, the coupling of the two models requires that the temporal multiscale nature of the problem is properly addressed. This is particularly important in the design of a framework to compute the model predictions. To address this problem, we propose an iterative time integration algorithm to efficiently solve the combined system, which is one of the novelties of our contribution.

\section{Materials and methods}

In this section, we first introduce the metabolic and electrophysiological mathematical models which are the building blocks of our combined model, then we describe how they are coupled.

The cerebral domain that we investigate is modeled as a four-compartment model comprising two cellular compartments, astrocyte and neuron, plus the surrounding extracellular space (ECS) that, together, constitute the brain tissue 
compartment. The ECS interfaces with the blood compartment via the blood-brain barrier, through which the tissue compartment exchanges some metabolites with the blood.

Each cellular compartment is equipped with its own internal metabolic network and the biochemical communication between the two compartments occurs through the ECS. We assume that the metabolic processes in the two cells are driven by their energetic needs and produce the ATP required for household and signaling functions.

The electrophysiological model is an extension of the classical Hodgin-Huxley model that describes the time course of the membrane voltage potential, the gating functions, and the concentrations of electrolytes in the cell compartments and in ECS. The coupling with the metabolism in the cells ensures that enough ATP is available to keep the ion pump functions active.

Our electrophysiology model is driven by a stimulus function $\xi(t), t=$ time, describing an exogenous neuronal signal incoming from outside the unit. The input of the metabolic system comprises the blood flow $q(t)$, and the arterial concentrations of the metabolites. A schematic picture of the structure of the electro-metabolic complex is shown in Figure 1. The details and governing equations of each model are presented in the next subsections.

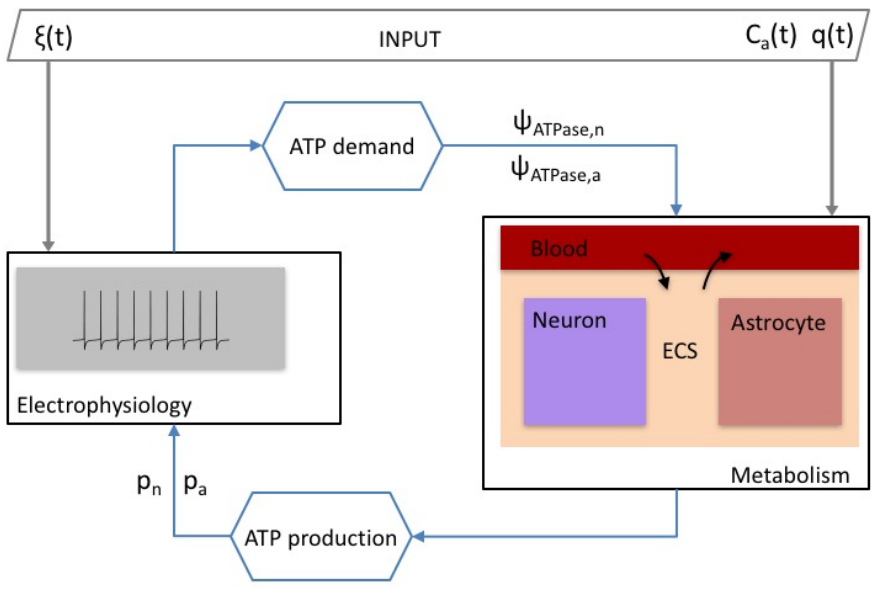

Figure 1: A schematic representation of a unit of the electro-metabolic model, highlighting the coupling of the two dynamics through the demand/supply chain of ATP production; $p_{n}$ and $p_{a}$ denote the phosphorylation states of neuron and astrocyte, respectively, while $\psi_{\text {ATPase,n }}$ and $\psi_{\text {ATPase,a }}$ are the ATP dephosphorylation fluxes in neuron and astrocyte. The stimulus function $\xi(t)$ is the input for the electrophysiology subunit and governs the frequency of the neuronal activation, accounting for exogenous neuronal signal incoming from outside the unit. The blood flow $q(t)$ and the arterial concentration of metabolites $C_{a}(t)$ are the external input for the metabolic subunit.

\subsection{Metabolic multi-compartment model}

The metabolism of the neuron-astrocyte complex is described as a multi-compartment well-mixed model comprising four separate compartments: blood, extracellular space (ECS), neuron, and astrocyte. Convection in the blood compartment carries the metabolites that enter the ECS and are subsequently taken into the neuron and astrocyte, the only two compartments where reactions can occur. For completeness, a brief overview of the four compartments is provided below. The dynamic equations describing the rate of change of the metabolites over time are scaled to correspond to a unit volume: the volume fractions of each compartment are denoted by $\eta_{\mathrm{x}}, \mathrm{x} \in\{\mathrm{b}, \mathrm{ECS}, \mathrm{n}, \mathrm{a}\}$ with self-explanatory indexing. We assume that the tissue volume fractions sum up to a unity. 


\subsubsection{Blood}

Following what is by now a rather established procedure, our simplified metabolic model follows three substances in the blood compartment: glucose (Glc), lactate (Lac) and oxygen $\left(\mathrm{O}_{2}\right)$. We denote the time dependent blood flow by $q=q(t)$ and the constant mixing ratio of arterial and venous blood in the blood compartment by $F, 0<F<1$. The concentrations [ ] of glucose and lactate in blood satisfy the differential equations

$$
\eta_{\mathrm{b}} \frac{d[\mathrm{X}]_{\mathrm{b}}}{d t}=\frac{q}{F}\left([\mathrm{X}]_{\mathrm{a}}-[\mathrm{X}]_{\mathrm{b}}\right)-J_{\mathrm{X}}, \quad \mathrm{X} \in\left\{\text { Glc, Lac, } \mathrm{O}_{2}\right\}
$$

where []$_{\mathrm{a}}$ denotes the presumably known arterial concentration and $J_{\mathrm{X}}$ the flux of the metabolite between blood and ECS, with the sign convention that $J_{\mathrm{X}}>0$ indicates net flux from blood to ECS. These transfer rates depend on the concentrations in blood and ECS, and to account for their facilitated nature we use symmetric Michaelis-Menten expressions

$$
J_{\mathrm{X}}=T_{\mathrm{b}, \mathrm{X}}\left(\frac{[\mathrm{X}]_{\mathrm{b}}}{K_{b, \mathrm{X}}+[\mathrm{X}]_{\mathrm{b}}}-\frac{[\mathrm{X}]_{\mathrm{ECS}}}{K_{\mathrm{b}, \mathrm{X}}+[\mathrm{X}]_{\mathrm{ECS}}}\right),
$$

where $T_{\mathrm{b}, \mathrm{X}}$ is the maximum transport rate, $K_{\mathrm{b}, \mathrm{X}}$ is the affinity constant, and $[\mathrm{X}]_{\mathrm{ECS}}$ is the metabolite concentration in ECS.

The equation that describes the dynamics of oxygen needs to be modified to account for the fact that oxygen can be present freely dissolved in the plasma, and bound to hemoglobin. We define the total oxygen concentration, denoted simply by $\left[\mathrm{O}_{2}\right]_{\mathrm{b}}$ in terms of the free oxygen concentration $\left[\mathrm{O}_{2}\right]_{\mathrm{b} \text {,free }}$ according to Hill's equation ([31]),

$$
\left[\mathrm{O}_{2}\right]_{\mathrm{b}}=\left[\mathrm{O}_{2}\right]_{\mathrm{b}, \text { free }}+4 \mathrm{Hct}[\mathrm{Hb}] \frac{\left[\mathrm{O}_{2}\right]_{\mathrm{b}, \text { free }}^{n}}{K_{\mathrm{H}}^{n}+\left[\mathrm{O}_{2}\right]_{\mathrm{b}, \text { free }}^{n}}=H\left(\left[\mathrm{O}_{2}\right]_{\mathrm{b}, \text { free }}\right),
$$

where Hct is the hematocrit, $[\mathrm{Hb}]$ the hemoglobin concentration in plasma, Hill's constant is set to $n=5 / 2$ and $K_{\mathrm{H}}$ is the corresponding affinity constant. The dynamic model for the blood concentration of oxygen is written in terms of the total oxygen concentration, where the transport flux obeys a modified Fick's law for the free concentration,

$$
J_{\mathrm{O}_{2}}=\lambda_{\mathrm{b}, \mathrm{O}_{2}}\left(\left[\mathrm{O}_{2}\right]_{\mathrm{b}, \text { free }}-\left[\mathrm{O}_{2}\right]_{\mathrm{ECS}}\right)^{\kappa}=\lambda_{\mathrm{b}, \mathrm{O}_{2}}\left(H^{-1}\left(\left[\mathrm{O}_{2}\right]_{\mathrm{b}}\right)-\left[\mathrm{O}_{2}\right]_{\mathrm{ECS}}\right)^{\kappa}, \quad \kappa=0.1 .
$$

With this modification the model is capable to reproduce the experimentally observed behavior of the Oxygen Glucose Index (OGI) during the neuronal activation [21]. A plausible physiological justification for this choice is presented in the Discussion section.

We collect the concentrations of all metabolites in the blood domain in a vector,

$$
C_{\mathrm{b}}(t)=\left[\begin{array}{c}
{[\mathrm{Glc}]_{\mathrm{b}}(t)} \\
{[\mathrm{Lac}]_{\mathrm{b}}(t)} \\
{\left[\mathrm{O}_{2}\right]_{\mathrm{b}}(t)}
\end{array}\right]
$$

and list all parameters related to the blood model in Table 1.

\begin{tabular}{|cc|ccc|ccc|}
\hline \multicolumn{2}{|c|}{ Volume fractions } & \multicolumn{3}{|c|}{ Blood Flow parameters } & \multicolumn{3}{c|}{ Arterial concentrations } \\
\hline Parameter & Value & Parameter & Value & Units & Parameter & Value & Units \\
\hline$\eta_{n}$ & 0.4 & Hct & 0.45 & & $C_{\mathrm{a}, \mathrm{Glc}}$ & 5 & $\mathrm{mM}$ \\
$\eta_{a}$ & 0.3 & $\mathrm{Hb}$ & 5.18 & & $C_{\mathrm{a}, \mathrm{Lac}}$ & 1.1 & $\mathrm{mM}$ \\
$\eta_{\text {ecs }}$ & 0.3 & $\mathrm{~K}_{\mathrm{H}}$ & $36.4 \cdot 10^{-3}$ & $\mathrm{mM}$ & $C_{\mathrm{a}, \mathrm{O}}$ & 9.14 & $\mathrm{mM}$ \\
$\eta_{b}$ & 0.04 & $Q$ & 0.40 & $\mathrm{~mL} / \mathrm{min}$ & & & \\
\hline
\end{tabular}

Table 1: Volume fractions and blood related parameters: Volume fractions of the four compartments (left), values of the parameters in the expression for blood flow (center) and arterial concentrations of glucose, lactate and oxygen (right). 


\subsubsection{Extracellular space}

In extracellular space, we follow the same metabolites as in blood: glucose, lactate and oxygen. Since no reactions take place in this compartment, and consequently, the concentrations change only through exchanges of the substrates with adjacent compartments, therefore the governing equations are of the form

$$
\eta_{\mathrm{ECS}} \frac{d[\mathrm{X}]_{\mathrm{ECS}}}{d t}=J_{\mathrm{X}}-j_{\mathrm{X}}^{\mathrm{n}}-j_{\mathrm{X}}^{\mathrm{a}}, \quad \mathrm{X} \in\left\{\mathrm{Glc}, \mathrm{Lac}, \mathrm{O}_{2}\right\}
$$

where $j_{\mathrm{X}}^{\mathrm{n}}$ and $j_{\mathrm{X}}^{\mathrm{a}}$ are the transport rates from ECS to neuron and astrocyte, respectively, with the sign convention that positive flux is directed away from ECS. The transport of glucose and lactate into the cells is mediated by the GLUT and MCT transporters, and the relative rates are expressed in Michaelis-Menten form,

$$
j_{\mathrm{X}}^{\mathrm{c}}=T_{\mathrm{c}, \mathrm{X}}\left(\frac{[\mathrm{X}]_{\mathrm{ECS}}}{K_{\mathrm{c}, \mathrm{X}}+[\mathrm{X}]_{\mathrm{ECS}}}-\frac{[\mathrm{X}]_{\mathrm{c}}}{K_{\mathrm{c}, \mathrm{X}}+[\mathrm{X}]_{\mathrm{c}}}\right), \quad \mathrm{c} \in\{\mathrm{n}, \mathrm{a}\},
$$

while the oxygen transport obeys Fick's law with constant permeabilities,

$$
j_{\mathrm{O}_{2}}^{\mathrm{c}}=\lambda_{\mathrm{c}, \mathrm{O}_{2}}\left(\left[\mathrm{O}_{2}\right]_{\mathrm{ECS}}-\left[\mathrm{O}_{2}\right]_{\mathrm{c}}\right), \quad \mathrm{c} \in\{\mathrm{n}, \mathrm{a}\} .
$$

As in the blood domain, we collect the concentrations into a vector,

$$
C_{\mathrm{ECS}}(t)=\left[\begin{array}{c}
{[\mathrm{Glc}]_{\mathrm{ECS}}(t)} \\
{[\mathrm{Lac}]_{\mathrm{ECS}}(t)} \\
{\left[\mathrm{O}_{2}\right]_{\mathrm{ECS}}(t)}
\end{array}\right] .
$$

The values of the parameters for the transport fluxes are collected in Table 2.

\begin{tabular}{|c|lll|lcl|llll|}
\hline \multirow{2}{*}{ Flux } & \multicolumn{3}{|c|}{ Blood $\leftrightarrow$ ECS } & \multicolumn{3}{c|}{ ECS $\leftrightarrow$ Neuron } & \multicolumn{3}{c|}{ ECS $\leftrightarrow$ Astrocyte } \\
\cline { 2 - 10 } & Parameter & Value & Units & Parameter & Value & Units & Parameter & Value & Units \\
\hline \multirow{2}{*}[\mathrm{Glc}]{} & $T_{\mathrm{b}, \mathrm{Glc}}$ & 0.02 & $\mathrm{mM} / \mathrm{s}$ & $T_{\mathrm{n}, \mathrm{Glc}}$ & 83.33 & $\mathrm{mM} / \mathrm{s}$ & $T_{\mathrm{a}, \mathrm{Glc}}$ & 83.33 & $\mathrm{mM} / \mathrm{s}$ \\
& $K_{\mathrm{b}, \mathrm{Glc}}$ & 4.60 & $\mathrm{mM}$ & $K_{\mathrm{n}, \mathrm{Glc}}$ & 5.00 & $\mathrm{mM}$ & $K_{\mathrm{a}, \mathrm{Glc}}$ & 12500.00 & $\mathrm{mM}$ \\
\hline \multirow{2}{*}[\mathrm{Lac}]{} & $T_{\mathrm{b}, \mathrm{Lac}}$ & 0.17 & $\mathrm{mM} / \mathrm{s}$ & $T_{\mathrm{n}, \mathrm{Lac}}$ & 66.67 & $\mathrm{mM} / \mathrm{s}$ & $T_{\mathrm{a}, \mathrm{Lac}}$ & 66.67 & $\mathrm{mM} / \mathrm{s}$ \\
& $K_{\mathrm{b}, \mathrm{Lac}}$ & 5.00 & $\mathrm{mM}$ & $K_{\mathrm{n}, \mathrm{Lac}}$ & 0.40 & $\mathrm{mM}$ & $K_{\mathrm{a}, \mathrm{Lac}}$ & 0.40 & $\mathrm{mM}$ \\
\hline$[\mathrm{O}]_{2}$ & $\lambda_{\mathrm{b}, \mathrm{O}_{2}}$ & 0.04 & $1 / \mathrm{s}$ & $\lambda_{\mathrm{n}, \mathrm{O}_{2}}$ & 0.94 & $1 / \mathrm{s}$ & $\lambda_{\mathrm{a}, \mathrm{O}_{2}}$ & 0.68 & $1 / \mathrm{s}$ \\
\hline
\end{tabular}

Table 2: Transports: List of the relevant parameter in the mathematical expression of the transport rate of the three metabolites exchanged between compartments and the values used in the computed examples. $T_{c, m}$ is the maximum transport rate and $K_{c, m}$ the affinity constant in the Michaelis-Menten expression for the transport rate of metabolite $m$ from compartment $c$, where $c=$ blood, neuron, astrocyte or ECS, $m=$ glucose or lactate and $\lambda_{c, O_{2}}$ is the parameter in Fick's law describing the diffusion of oxygen from compartment $c$.

\subsubsection{Neuron and astrocyte}

Changes in the concentrations of substances in the cell domains may occur because of transport to and from the extracellular space and reaction taking place.

In the cell compartments, we follow the time course of the concentrations of the ten metabolites listed in Table 3. which participate in the chemical reactions included in the present simplified model and listed in Table 4. Of these metabolites, only glucose, lactate and oxygen are transported through the cell membrane. 


\begin{tabular}{c|cccccccccc} 
& {$[\mathrm{Glc}]$} & {$\left[\mathrm{O}_{2}\right]$} & {$[\mathrm{Lac}]$} & {$[\mathrm{Pyr}]$} & {$[\mathrm{PCr}]$} & {$[\mathrm{Cr}]$} & {$[\mathrm{ATP}]$} & {$[\mathrm{ADP}]$} & {$[\mathrm{NADH}]$} & {$[\mathrm{NAD}]$} \\
\hline $\mathrm{B}$ & 4.51 & 6.67 & 1.24 & & & & & & & \\
$\mathrm{E}$ & 1.19 & 0.04 & 1.30 & & & & & & & \\
$\mathrm{~A}$ & 0.65 & 0.03 & 1.30 & 0.35 & 10.32 & $1.1 \mathrm{e}-3$ & 2.17 & 0.03 & $1.2 \mathrm{e}-3$ & 0.03 \\
$\mathrm{~N}$ & 1.19 & 0.03 & 1.30 & 0.38 & 10.33 & $3.0 \mathrm{e}-4$ & 2.18 & $6.3 \mathrm{e}-3$ & $1.2 \mathrm{e}-3$ & 0.03
\end{tabular}

Table 3: Metabolites: The column are labelled by the metabolites and the rows by the 4 compartments (blood (B), extracellular space (E), astrocyte (A) and Neuron $(\mathrm{N})$. The numerical entries in the table are the resting concentrations (in $\mathrm{mM}$ ) of the metabolite corresponding to the column in the compartment corresponding to the row.

Letting $\mathrm{S}$ denote the stoichiometric matrix with entries

$$
s_{\mathrm{X}, \mathrm{R}}=\# \text { of molecules of } \mathrm{X} \text { produced/depleted by reaction } \mathrm{R},
$$

where a positive sign indicates production and a negative one depletion, the mass balance equation for metabolites $\mathrm{X}$ in the cellular compartment $\mathrm{c}$ can be written as

$$
\eta_{\mathrm{c}} \frac{d[\mathrm{X}]_{\mathrm{c}}}{d t}=j_{\mathrm{X}}^{\mathrm{c}}+\sum_{\mathcal{R}} s_{\mathrm{X}, \mathrm{R}} \psi_{\mathrm{R}}^{\mathrm{c}}, \quad \mathrm{c}=\mathrm{n}, \mathrm{a}
$$

where the transport rate $j_{\mathrm{X}}^{\mathrm{c}}$ is modeled according to (4) for glucose and lactate, according to (5) for oxygen, and vanishes for all other substances. The reaction rates $\psi_{\mathrm{R}}^{\mathrm{c}}$ appearing in the second term are concentration dependent, and their explicit expressions and parameter values are listed in Table 5, where we use the notations

$$
p_{\mathrm{n}}=\frac{[\mathrm{ATP}]_{\mathrm{n}}}{[\mathrm{ADP}]_{\mathrm{n}}} \quad p_{\mathrm{a}}=\frac{[\mathrm{ATP}]_{\mathrm{a}}}{[\mathrm{ADP}]_{\mathrm{a}}}
$$

for the phosphorylation states of the two cells, and

$$
r_{\mathrm{n}}=\frac{[\mathrm{NADH}]_{\mathrm{n}}}{\left[\mathrm{NAD}^{+}\right]_{\mathrm{n}}} \quad r_{\mathrm{a}}=\frac{[\mathrm{NADH}]_{\mathrm{a}}}{\left[\mathrm{NAD}^{+}\right]_{\mathrm{a}}}
$$

\begin{tabular}{|c|c|c|c|}
\hline Name & Neuron & Astrocyte & Reaction \\
\hline Gcl & $\psi_{\mathrm{Gcl}, \mathrm{n}}$ & $\psi_{\mathrm{Gcl}, \mathrm{a}}$ & $\mathrm{Glc}^{2} \mathrm{NAD}^{+}+2 \mathrm{ADP} \longrightarrow 2 \mathrm{Pyr}+2 \mathrm{NADH}+2 \mathrm{ATP}$ \\
\hline LDH1 & $\psi_{\mathrm{LDH} 1, \mathrm{n}}$ & $\psi_{\mathrm{LDH} 1, \mathrm{a}}$ & $\mathrm{Pyr}+\mathrm{NADH} \longrightarrow \mathrm{Lac}+\mathrm{NAD}^{+}$ \\
\hline LDH2 & $\psi_{\mathrm{LDH} 2, \mathrm{n}}$ & $\psi_{\mathrm{LDH} 2, \mathrm{a}}$ & $\mathrm{Lac}+\mathrm{NAD}^{+} \longrightarrow \mathrm{Pyr}+\mathrm{NADH}$ \\
\hline TCA & $\psi_{\mathrm{TCA}, \mathrm{n}}$ & $\psi_{\mathrm{TCA}, \mathrm{a}}$ & $\mathrm{Pyr}+\mathrm{ADP}+5 \mathrm{NAD}^{+} \longrightarrow 3 \mathrm{CO}_{2}+\mathrm{ATP}+5 \mathrm{NADH}$ \\
\hline OxPhos & $\psi_{\mathrm{OxPhos}, \mathrm{n}}$ & $\psi_{\mathrm{OxPhos}, \mathrm{a}}$ & $\mathrm{O}_{2}+2 \mathrm{NADH}+5 \mathrm{ADP} \longrightarrow 2 \mathrm{NAD}^{+}+5 \mathrm{ATP}+2 \mathrm{H}_{2} \mathrm{O}$ \\
\hline $\mathrm{PCr}$ & $\psi_{\mathrm{PCr}, \mathrm{n}}$ & $\psi_{\mathrm{PCr}, \mathrm{a}}$ & $\mathrm{PCr}+\mathrm{ADP} \longrightarrow \mathrm{Cr}+\mathrm{ATP}$ \\
\hline $\mathrm{Cr}$ & $\psi_{\mathrm{Cr}, \mathrm{n}}$ & $\psi_{\mathrm{Cr}, \mathrm{a}}$ & $\mathrm{Cr}+\mathrm{ATP} \longrightarrow \mathrm{PCr}+\mathrm{ADP}$ \\
\hline ATPase & $\psi_{\text {ATPase,n }}$ & $\psi_{\text {ATPase,a }}$ & $\mathrm{ATP} \rightarrow \mathrm{ADP}$ \\
\hline
\end{tabular}

for the redox states. The ATPase reaction rate is not included in the list because of its special role in the coupling with the electrophysiology model.

Table 4: Reactions: List of the lumped reactions included in the model. Abbreviations for the reaction names: $\mathrm{Gcl}=$ Glycolysis, $\mathrm{LDH}=$ Lactate dehydrogenase, reversible reaction, TCA $=$ Tricarboxylic acid cycle, OxPhos $=$ Oxidative phosphorylation, $\mathrm{PCr}=$ Phosphocreatine dephosphorylation, $\mathrm{Cr}=$ Creatine phosphorylation, ATPase $=$ ATP dephosphorylation, mostly accounting for the $\mathrm{Na}^{+} / \mathrm{K}^{+}$ATPase. 


\begin{tabular}{|c|c|c|c|c|}
\hline Reaction & Parameter & Units & Neuron & Astrocyte \\
\hline \multirow[t]{2}{*}{ Glycolysis $\left(\psi_{\mathrm{Gcl}}\right)$} & $V_{\mathrm{Gcl}}$ & $\mathrm{mM} / \mathrm{s}$ & 0.26 & 0.25 \\
\hline & $K_{\mathrm{Gcl}}$ & $\mathrm{mM}$ & 4.60 & 3.10 \\
\hline \multirow{2}{*}{$V_{\mathrm{Gcl}} \frac{1 / p}{\mu_{\mathrm{Gcl}}+1 / p} \frac{1 / r}{\nu_{\mathrm{Gcl}}+1 / r} \frac{[\mathrm{Glc}]}{[\mathrm{Glc}]+K_{\mathrm{Gcl}}}}$, & $\mu_{\mathrm{Gcl}}$ & & 0.09 & 0.09 \\
\hline & $\nu_{\mathrm{Gcl}}$ & & 10.00 & 10.00 \\
\hline Lactate dehydrogenase $\left(\psi_{\mathrm{LDH} 1}\right)$ & $V_{\mathrm{LDH} 1}$ & $\mathrm{mM} / \mathrm{s}$ & 1436.00 & 4160.00 \\
\hline \multirow{2}{*}{$V_{\mathrm{LDH} 1} \frac{r}{\nu_{\mathrm{LDH} 1}+r} \frac{[\mathrm{Pyr}]}{[\mathrm{Pyr}]+K_{\mathrm{LDH} 1}}$} & $K_{\mathrm{LDH} 1}$ & $\mathrm{mM}$ & 2.15 & 6.24 \\
\hline & $\nu_{\mathrm{LDH} 1}$ & & 0.10 & 0.10 \\
\hline Lactate dehydrogenase $\left(\psi_{\mathrm{LDH} 2}\right)$ & $V_{\mathrm{LDH} 2}$ & $\mathrm{mM} / \mathrm{s}$ & 1579.83 & 3245.00 \\
\hline \multirow{2}{*}{$V_{\mathrm{LDH} 2} \frac{1 / r}{\nu_{\mathrm{LDH} 2}+1 / r} \frac{[\mathrm{Lac}]}{[\mathrm{Lac}]+K_{\mathrm{LDH} 2}}$} & $K_{\mathrm{LDH} 2}$ & $\mathrm{mM}$ & 23.70 & 48.66 \\
\hline & $\nu_{\mathrm{LDH} 2}$ & & 10.00 & 10.00 \\
\hline TCA cycle $\left(\psi_{\text {TCA }}\right)$ & $V_{\mathrm{TCA}}$ & $\mathrm{mM} / \mathrm{s}$ & 0.03 & 0.01 \\
\hline \multirow{3}{*}{$V_{\mathrm{TCA}} \frac{1 / p}{\mu_{\mathrm{TCA}}+1 / p} \frac{1 / r}{\nu_{\mathrm{TCA}}+1 / r} \frac{[\mathrm{Pyr}]}{[\mathrm{Pyr}]+K_{\mathrm{TCA}}}$} & $K_{\mathrm{TCA}}$ & $\mathrm{mM}$ & 0.01 & 0.01 \\
\hline & $\mu_{\mathrm{TCA}}$ & & 0.01 & 0.01 \\
\hline & $\nu_{\mathrm{TCA}}$ & & 10.00 & 10.00 \\
\hline \multirow[t]{2}{*}{ Oxidative phosphorylation $\left(\psi_{\mathrm{OxPhos}}\right)$} & $V_{\text {OxPhos }}$ & $\mathrm{mM} / \mathrm{s}$ & 8.18 & 2.55 \\
\hline & $K_{\text {OxPhos }}$ & $\mathrm{mM}$ & 1.00 & 1.00 \\
\hline \multirow{2}{*}{$V_{\text {OxPhos }} \frac{1 / p}{\mu_{\text {OxPhos }}+1 / p} \frac{r}{\nu_{\text {OxPhos }}+r} \frac{\left[\mathrm{O}_{2}\right]}{\left[\mathrm{O}_{2}\right]+K_{\text {OxPhos }}}$} & $\mu_{\mathrm{LDH} 1}$ & & 0.01 & 0.01 \\
\hline & $\nu_{\mathrm{LDH1}}$ & & 0.10 & 0.10 \\
\hline \multirow{3}{*}{$\begin{array}{l}\text { Creatine phosphorylation }\left(\psi_{\mathrm{Cr}}\right) \\
V_{\mathrm{Cr}} \frac{p}{\mu_{\mathrm{Cr}}+p} \frac{[\mathrm{Cr}]}{[\mathrm{Cr}]+K_{\mathrm{Cr}}}\end{array}$} & $V_{\mathrm{Cr}}$ & $\mathrm{mM} / \mathrm{s}$ & 16666.67 & 16666.67 \\
\hline & $K_{\mathrm{Cr}}$ & $\mathrm{mM}$ & 495.00 & 495.00 \\
\hline & $\mu_{\mathrm{Cr}}$ & & 0.01 & 0.01 \\
\hline \multirow{3}{*}{$\begin{array}{l}\text { Creatine dephosphorylation }\left(\psi_{\mathrm{PCr}}\right) \\
V_{\mathrm{PCr}} \frac{1 / p}{\mu_{\mathrm{PCr}}+1 / p} \frac{[\mathrm{PCr}]}{[\mathrm{PCr}]+K_{\mathrm{PCr}}}\end{array}$} & $V_{\mathrm{PCr}}$ & $\mathrm{mM} / \mathrm{s}$ & 16666.67 & 16666.67 \\
\hline & $K_{\mathrm{PCr}}$ & $\mathrm{mM}$ & 528.00 & 528.00 \\
\hline & $\mu_{\mathrm{PCr}}$ & & 100.00 & 100.00 \\
\hline
\end{tabular}

Table 5: Reactions rates: Michaelis-Menten type expressions for the reaction fluxes in neuron and astrocyte and the values of the respective parameters.

We collect the concentrations of the ten metabolites tracked in neuron and astrocyte into the vectors $C_{\mathrm{n}}(t), C_{\mathrm{a}}(t) \in$ $\mathbb{R}^{10}$.

As a final remark, we point out that the present model does not explicitly describe the glutamate-glutamine cycle. Unlike in the articles $[13,14]$, where the energetic coupling of the metabolic activity of the two cells was effectuated by lumping an overall implicit energetic cost with the neurotransmitter cycle, in the present model, the energetic cost will be tied in a more physiologically correct manner directly to the ion traffic, as explained in the next section.

\subsubsection{Metabolic model}

After collecting the concentrations of metabolites in the different compartments into the vector

$$
C(t)=\left[\begin{array}{c}
C_{\mathrm{b}}(t) \\
C_{\mathrm{ECS}}(t) \\
C_{\mathrm{n}}(t) \\
C_{\mathrm{a}}(t)
\end{array}\right] \in \mathbb{R}^{26},
$$

and introducing the diagonal mass matrix $M$

$$
\mathrm{M}=\operatorname{diag}\left(\eta_{\mathrm{b}} \mathrm{I}_{3}, \eta_{\mathrm{ECS}} \mathrm{I}_{3}, \eta_{\mathrm{n}} \mathrm{I}_{10,}, \eta_{\mathrm{a}} \mathrm{I}_{10}\right) \in \mathbb{R}^{26 \times 26},
$$

where $\mathrm{I}_{k}$ is an identity of size $k \times k$, we can write the governing equations of the metabolic model compactly in the form

$$
\mathrm{M} \frac{d C}{d t}=F\left(C, C_{\mathrm{a}}, \psi_{\text {ATPase }, \mathrm{n}}, \psi_{\text {ATPase }, \mathrm{a}}, q\right)
$$


where the dependency of the right hand side on ATP dephosphorylation is highlighted to emphasize its special role. Note that the reaction fluxes of the ATP dephosphorylation $\psi_{\text {ATPase, }}(t)$ and $\psi_{\text {ATPase, }}(t)$ in neuron and astrocyte are not specified in terms of the metabolite concentrations.

The arterial concentrations $C_{\mathrm{a}} \in \mathbb{R}^{3}$ and the blood flow $q(t)$ serve as input for the system, while the dephosphorylation fluxes are the link between metabolism and electrophysiology, as will be described next.

\subsection{Electrophysiology model}

Our electrophysiology model is a modification of the classical Hodgkin-Huxley (HH) model for the neuron membrane potential, augmented with a potassium dynamics that takes into account the astrocytic potassium cleaning and diffusion, as described in $[17,7]$. The differential equations for the voltage potential of the neuronal membrane and for the gating functions $m, h$ and $n$ are of the form

$$
\begin{aligned}
c_{m} \frac{d V}{d t} & =-I_{\mathrm{Na}^{+}}-I_{\mathrm{K}^{+}}-I_{\text {leak }}, \\
\frac{d w}{d t} & =\varphi\left(\alpha_{w}(V)(1-w)-\beta_{w}(V) w\right), \quad w \in\{h, n, m\},
\end{aligned}
$$

where $c_{m}>0$ is the membrane capacitance. The ionic currents of sodium $\left(\mathrm{Na}^{+}\right)$, potassium $\left(\mathrm{K}^{+}\right)$and the leak current of chloride, which are a slight modification of those proposed in the $\mathrm{HH}$ model, are of the form

$$
\begin{aligned}
I_{\mathrm{Na}^{+}} & =g_{\mathrm{Na}^{+}} m^{3} h\left(V-V_{\mathrm{Na}^{+}}\right)+g_{\mathrm{Na}^{+}, \text {leak }}\left(V-V_{\mathrm{Na}^{+}}^{+}\right), \\
I_{\mathrm{K}^{+}} & =g_{\mathrm{K}^{+}} n^{4}\left(V-V_{\mathrm{K}^{+}}\right)+g_{\mathrm{K}^{+}, \text {leak }}\left(V-V_{\mathrm{K}^{+}}\right), \\
I_{\text {leak }, \mathrm{Cl}^{-}} & =g_{\mathrm{Cl}^{-}}\left(V-V_{\mathrm{Cl}^{-}}\right)
\end{aligned}
$$

where $g_{\mathrm{Na}^{+}}, g_{\mathrm{Na}^{+}, \text {leak }}, g_{\mathrm{K}^{+}}$and $g_{\mathrm{K}^{+}, \text {leak }}$ are the conductances and leak conductances of sodium and potassium, respectively, and $g_{\mathrm{Cl}^{-}}$is the leak conductance of chloride. The values used in the simulations are given in Table 6.

\begin{tabular}{|c|c|c|c|}
\hline Parameter description & Symbol & Value & Unit \\
\hline Sodium conductance & $g_{\mathrm{Na}^{+}}$ & 100 & $\mathrm{mS} / \mathrm{cm}^{2}$ \\
Potassium conductance & $g_{\mathrm{K}^{+}}$ & 40 & $\mathrm{mS} / \mathrm{cm}^{2}$ \\
Chloride conductance & $g_{\mathrm{Cl}^{-}}$ & 0.05 & $\mathrm{mS} / \mathrm{cm}^{2}$ \\
Sodium leak conductance & $g_{\mathrm{Na}^{+}, \text {leak }}^{0}$ & 0.0175 & $\mathrm{mS} / \mathrm{cm}^{2}$ \\
Potassium leak conductance & $g_{\mathrm{K}^{+}, \text {leak }}$ & 0.05 & $\mathrm{mS} / \mathrm{cm}^{2}$ \\
Capacitance & $C$ & 1 & $\mu \mathrm{F} / \mathrm{cm}^{2}$ \\
Time constant & $\varphi$ & 3 & $1 / \mathrm{ms}$ \\
\hline
\end{tabular}

Table 6: Parameters in the electrophysiologic model: ion conductances, membrane capacitance and time constant.

The reversal potentials $V_{\mathrm{Na}^{+}}, V_{\mathrm{K}^{+}}$and $V_{\mathrm{Cl}^{-}}$which depend on the ion concentrations inside and outside the membrane, are described via the Nerst equations

$$
V_{\mathrm{X}}=26.64 \ln \left(\frac{[\mathrm{X}]_{\mathrm{o}}}{[\mathrm{X}]_{\mathrm{i}}}\right), \quad \mathrm{X} \in\left\{\mathrm{Na}^{+}, \mathrm{K}^{+}, \mathrm{Cl}^{-}\right\}
$$

Since our model does not follow the chloride dynamics, we keep the values of its concentration inside and outside the cell fixed at $\left[\mathrm{Cl}^{-}\right]_{\mathrm{o}}=6 \mathrm{mM}$ and $\left[\mathrm{Cl}^{-}\right]_{\mathrm{i}}=130 \mathrm{mM}$, respectively. Following the model in [17], the exterior sodium and interior potassium concentrations are assumed to be determined by the interior sodium concentration through the algebraic equations

$$
\begin{aligned}
{\left[\mathrm{Na}^{+}\right]_{\mathrm{o}} } & =144 \mathrm{mM}-\beta\left(\left[\mathrm{Na}^{+}\right]_{\mathrm{i}}-11.5 \mathrm{mM}\right) \\
{\left[\mathrm{K}^{+}\right]_{\mathrm{i}} } & =140 \mathrm{mM}+\left(11.5 \mathrm{mM}-\left[\mathrm{Na}^{+}\right]_{\mathrm{i}}\right),
\end{aligned}
$$


where $11.5 \mathrm{mM}$ and $140 \mathrm{mM}$ are the inner concentrations at rest of sodium and potassium, $144 \mathrm{mM}$ is the outer resting concentration of sodium, $1 / \varphi>0$ is the time constant for the gating variables and the saturating functions $\alpha_{w}$ and $\beta_{w}$ specific for each gating variable, are specified in Table 7.

\begin{tabular}{|c|c|c|c|}
\hline$w$ & $m$ & $h$ & $n$ \\
\hline$\alpha_{w}(V)$ & $0.1 \frac{V+30}{1-\exp (-(V+30) / 10)}$ & $0.07 \exp (-(V+44) / 20)$ & $0.01 \frac{V+34}{1-\exp (-(V+34) / 10)}$ \\
$\beta_{w}(V)$ & $4 \exp (-(V+55) / 18)$ & $\frac{1}{1+\exp (-(V+14) / 10)}$ & $0.125 \exp (-(V+44) / 80)$ \\
\hline
\end{tabular}

Table 7: Gating variables: mathematical description as voltage-dependent saturation functions.

Following [17], we assume that $m=m(V)$ reaches the equilibrium value fast enough to justify the steady state approximation

$$
m=m(V)=\frac{\alpha_{m}(V)}{\alpha_{m}(V)+\beta_{m}(V)},
$$

thus allowing the elimination of one equation from the system.

Because one aim of the present study is to understand the metabolic response to different levels of synaptic activity and, vice versa, whether and how altered metabolism limits the range of synaptic activity, it is important to take into account the role that the neurotransmitter glutamate plays in the synaptic cleft. As the glutamate secreted by the presynaptic neuron is sensed by the postsynaptic glutamate receptors, the postsynaptic ion channels open, leading to an increased influx of sodium. Rather than modeling this mechanism by describing the recycling of glutamate, we implement it as a temporary increase in the sodium and potassium leak conductances in (10)-(11), writing

$$
\left(g_{\mathrm{Na}^{+}, \text {leak }}(t), g_{\mathrm{K}^{+}, \text {leak }}(t)\right)=(1+\xi(t))\left(g_{\mathrm{Na}^{+}, \text {leak }}^{0}, g_{\mathrm{K}^{+}, \text {leak }}^{0}\right),
$$

where $g_{\mathrm{Na}^{+}, \text {leak }}^{0}$ and $g_{\mathrm{K}^{+} \text {, leak }}^{0}$ are the constant resting values of the leaks, and $\xi(t) \geq 0$ is an activation function that models the effect of glutamate.

To connect the electrophysiology model with the energetics of the cells, we describe the sodium concentration inside the neuron and the potassium concentration outside by the differential equations

$$
\begin{aligned}
\tau \frac{d\left[\mathrm{Na}^{+}\right]_{\mathrm{i}}}{d t} & =-\gamma I_{\mathrm{Na}^{+}}-3 J_{\mathrm{pump}, \mathrm{Na}^{+}}, \\
\tau \frac{d\left[\mathrm{~K}^{+}\right]_{\mathrm{o}}}{d t} & =\gamma \beta I_{\mathrm{K}^{+}}-2 \beta J_{\mathrm{pump}, \mathrm{Na}^{+}}-J_{\mathrm{glia}, \mathrm{K}^{+}}-J_{\mathrm{diff}, \mathrm{K}^{+}},
\end{aligned}
$$

where $J_{\mathrm{pump}, \mathrm{Na}^{+}}$is the ion (mass) current induced by the sodium-potassium pump, $J_{\mathrm{glia}, \mathrm{K}^{+}}$the ion current induced by the astrocytic potassium cleaning and $J_{\text {diff, } \mathrm{K}^{+}}$accounts for the diffusion of potassium away from the neighborhood. The coefficient $\tau=1000$ is the conversion factor from seconds to milliseconds. The scaling parameter $\gamma$ that converts the electric current to mass flux in [17], is assigned the value $\gamma=0.0445 \mathrm{mM} \mathrm{cm}^{2} / \mu \mathrm{C}$ on the basis of a geometric argument, and $\beta=\eta_{\mathrm{n}} / \eta_{\mathrm{ECS}}$. By modifying the model in [17], the ion mass currents are defined here through the equations

$$
\begin{aligned}
J_{\text {pump }, \mathrm{Na}^{+}} & =\frac{p_{\mathrm{n}}}{\mu_{\text {pump }}+p_{\mathrm{n}}}\left(\frac{\rho}{\left.1+\exp \left(25-\left[\mathrm{Na}^{+}\right]_{\mathrm{i}}\right) / 3\right)}\right) \times\left(\frac{1}{1+\exp \left(5.5-\left[\mathrm{K}^{+}\right]_{\mathrm{o}}\right)}\right), \\
J_{\text {glia }, \mathrm{K}^{+}} & =\frac{p_{\mathrm{a}}}{\mu_{\text {glia }}+p_{\mathrm{a}}} \frac{G_{\text {glia }}}{1+\exp \left(\left(18-\left[\mathrm{K}^{+}\right]_{\mathrm{o}}\right) / 2.5\right)}, \\
J_{\text {diff }, \mathrm{K}^{+}} & =\varepsilon\left(\left[\mathrm{K}^{+}\right]_{\mathrm{o}}-k_{\infty}\right),
\end{aligned}
$$


where $p_{\mathrm{n}}=p_{\mathrm{n}}(t)$ and $p_{\mathrm{a}}=p_{\mathrm{a}}(t)$ are the phosphorylation states of the neuron and astrocyte, respectively, and $\mu_{\text {pump }}=0.1$ and $\mu_{\text {glia }}=0.1$ are affinity constants. These formulas describe the feedback mechanism from the metabolism to the electrophysiology. We remark that the astrocytic potassium flux is not assumed to be directly ATP dependent, but rather the model acknowledges the fact that potassium cleaning incurs a metabolic cost in astrocytes, the details not being fully understood [25]. If the cells are unable to keep up a phosphorylation state providing sufficient pump action, the sodium and potassium ion fluxes necessarily slow down, causing an imbalance of ion concentrations.

\begin{tabular}{|c|c|c|c|c|}
\hline $\mathrm{V}(\mathrm{mV})$ & {$\left[\mathrm{K}^{+}\right]_{\mathrm{o}}(\mathrm{mM})$} & {$\left[\mathrm{Na}^{+}\right]_{\mathrm{i}}(\mathrm{mM})$} & $n$ & $h$ \\
\hline-56.1999 & 6.2773 & 11.5604 & 0.1558 & 0.9002 \\
\hline
\end{tabular}

Table 8: Electrophysiology model: Initial conditions of membrane potential, ionic concentrations and gating variables.

Finally, collecting all dynamic variables into the vector

$$
u(t)=\left[\begin{array}{c}
V(t) \\
{\left[\mathrm{Na}^{+}\right]_{\mathrm{i}}(t)} \\
{\left[\mathrm{K}^{+}\right]_{\mathrm{o}}(t)} \\
n(t) \\
h(t)
\end{array}\right] \in \mathbb{R}^{5}
$$

we can combine equations (8), (9), (16) and (17) into the non-linear system

$$
\frac{d u}{d t}=f\left(u, p_{\mathrm{n}}, p_{\mathrm{a}}, \xi\right)
$$

where the input comprises the phosphorylation states and the stimulus function.

\subsection{A coupled electro-metabolic model}

Equations (18)-(19) describe how the metabolic state of the cells regulates the electrophysiology. Conversely, the signaling to the metabolic system about the energetic needs of the cell, is encoded via the energetic cost of the ion pump action. When the ATP dephosphorylation reaction ATP $\rightarrow$ ADP takes place, it releases the binding energy $\Delta E=30.5 \mathrm{~kJ} / \mathrm{mol}$, which can be used by the cell to maintain the ion pump actions. Since one ATPase can supply the energy required to extrude three sodium ions in exchange for the entering of two potassium ions, the stoichiometric relation

$$
\text { ATPase + ion pump : } \quad \mathrm{ATP}+3 \mathrm{Na}_{\mathrm{i}}^{+}+2 \mathrm{~K}_{\mathrm{o}}^{+} \rightarrow \mathrm{ADP}+3 \mathrm{Na}_{\mathrm{o}}^{+}+2 \mathrm{~K}_{\mathrm{i}}^{+},
$$

holds for both neuron and astrocyte. In line with the metabolic modeling paradigm, we may write the mass balance equations for intracellular sodium of neuron and for extracellular potassium as

$$
\begin{aligned}
\eta_{\mathrm{n}} \frac{d\left[\mathrm{Na}^{+}\right]_{\mathrm{i}}}{d t} & =-\gamma \eta_{\mathrm{n}} I_{\mathrm{Na}^{+}}-3 \psi_{\text {ATPase }, \mathrm{n}}, \\
\eta_{\mathrm{ECS}} \frac{d\left[\mathrm{~K}^{+}\right]_{0}}{d t} & =\gamma \eta_{\mathrm{n}} I_{\mathrm{K}^{+}}-2 \psi_{\text {ATPase } \mathrm{n}}-2 \psi_{\text {ATPase }, \mathrm{a}}-\varepsilon_{\mathrm{ECS}}\left(\left[\mathrm{K}^{+}\right]_{\mathrm{o}}-k_{\infty}\right),
\end{aligned}
$$

where $\varepsilon_{\mathrm{ECS}}=\eta_{\mathrm{ECS}} \varepsilon$ and $\varepsilon$ is the diffusion coefficient.

Denote by

$$
I_{\mathrm{Na}^{+}, \text {leak }}(t)=g_{\mathrm{Na}^{+}, \text {leak }}(t)\left(V-V_{N a}^{+}\right)
$$


the leak current of sodium induced by the increase of membrane permeability by the function $\xi(t)$. Letting $I_{\mathrm{Na}^{+}}^{0}$, leak denote the average sodium leak current during baseline activity, we model the sodium current occurring in conjunction with the neurotransmitter activity as

$$
I_{\mathrm{Na}^{+}, \text {act }}(t)= \begin{cases}I_{\mathrm{Na}^{+}, \text {leak }}(t)-I_{\mathrm{Na}^{+}, \text {leak }}^{0}, & \text { if } \xi(t)>0 \\ 0 & \text { if } \xi(t)=0\end{cases}
$$

and the concomitant flux of sodium ions as

$$
J_{\mathrm{Na}^{+}, \text {act }}=\gamma I_{\mathrm{Na}^{+}, \text {act }},
$$

where $\gamma$ is the conversion factor from electric currents to mass fluxes. In light of the above relation between glutamate release and $\mathrm{Na}^{+}$ion currents, we implicitly assume a glutamate-glutamine flux

$$
J_{\text {glu }}=\frac{\gamma}{\sigma} I_{\mathrm{Na}^{+}, \text {act }},
$$

where $\sigma$ accounts for the energetic cost of cycling glutamate-glutamine during synaptic activity. In setting the value of $\sigma$, we rely on the argument from [6] that:

1. Each glutamate molecule taken up by astrocyte and processed to glutamine costs approximately 2.33 ATP, of which 1.33 for the uptake and 1 for the processing. For comparison, the energetic cost of vesicle packing in neuron is only 0.33 ATP per glutamate molecule.

2. A vesicle contains about 4000 glutamate molecules.

3. The release in the synaptic cleft of the glutamate molecules in a vesicle triggers the opening of 15-200 nonNMDA (N-methyl-D-aspartate) dependent sodium channels whose mean open time is in the range of 0.6-1.4 milliseconds. On average, the release of one glutamate vesicle leads to the entry of $200000 \mathrm{Na}^{+}$ions in the postsynaptic neuron.

4. The presence of glutamate in the cleft causes the opening of NMDA-dependent channels for the duration of about 50 milliseconds, so that approximately $180000 \mathrm{Na}^{+}$and $10000 \mathrm{Ca}^{2+}$ ions enter per vesicle. The activation of the $\mathrm{Ca}^{2+} / \mathrm{Na}^{+}$pump for the removal of the calcium brings in an additional $30000 \mathrm{Na}^{+}$ions, thanks to the 3:1 sodium-calcium exchange. In total, the opening of each vesicle is followed by the entry of 210000 sodium molecules.

In summary, we assume that for each molecule of glutamate released in the synaptic cleft, the number of $\mathrm{Na}^{+}$ions entering the neuron is $\sigma \approx 103$.

Our model accounts for the energetic cost of the underlying glutamine recycling with the following mathematical expression for the ATP dephosphorylation fluxes in neuron and astrocyte

$$
\begin{aligned}
& \psi_{\text {ATPase }, \mathrm{n}}=\mathrm{H}_{1}+s\left(\eta_{\mathrm{n}} J_{\text {pump }, \mathrm{Na}^{+}}+0.33 \frac{\gamma}{\sigma} I_{\mathrm{Na}^{+}, \text {act }}\right) \\
& \psi_{\text {ATPase }, \mathrm{a}}=\mathrm{H}_{2}+s\left(\frac{\eta_{\mathrm{ECS}}}{2} J_{\mathrm{glia}, \mathrm{K}^{+}}+2.33 \frac{\gamma}{\sigma} I_{\mathrm{Na}^{+}, \text {act }}\right)
\end{aligned}
$$

where $H_{1}$ and $H_{2}$ denote the household energy necessary to perform routine tasks. The quantification of $H_{1}$ and $H_{2}$ continues to be a topic of debate and will be addressed in detail below. The role of the parameter $s>0$ is to compensate for considering different total volumes in the process of calibrating the individual electrophysiology and metabolism models. The conversion of electric currents into mass fluxes depends on the assumed geometries of the cells, including the surface area which is poorly known. A discussion of the rational for the selection of a value for this the parameter is included in the results section. 
Summarizing, we can write the governing equations of the combined electro-metabolic model formally as

$$
\begin{aligned}
\mathrm{M} \frac{d C}{d t} & =F\left(C, C_{\mathrm{a}}, \psi_{\text {ATPase }, \mathrm{n}}, \psi_{\text {ATPase }, \mathrm{a}}, q\right) \\
\frac{d u}{d t} & =f\left(u, p_{\mathrm{n}}, p_{\mathrm{a}}, \xi\right)
\end{aligned}
$$

to emphasize the input $C_{\mathrm{a}}(t), q(t)$, and $\xi(t)$, and the functions (23) - (24) and (6) concisely as

$$
\begin{aligned}
\left(\psi_{\text {ATPase }, \mathrm{n}}, \psi_{\text {ATPase }, \mathrm{a}}\right) & =\Theta(u), \\
\left(p_{\mathrm{n}}, p_{\mathrm{a}}\right) & =\theta(C),
\end{aligned}
$$

providing the coupling between the two models.

\section{Computational framework for multiple time scales}

The computation of the predictions of the combined model is very challenging because of the several orders of magnitude difference in the characteristic time scales of the electrophysiology and metabolism processes. We address the multiscale nature of the problem by using a much finer time discretization for the electrophysiology propagation than for the metabolic portion, taking care of synchronizing the values the coupling quantities at the end of each slow time step.

\subsection{The slow time scale of the metabolic processes}

The model describes how the system evolves in time by tracking the concentrations of the metabolites. Since the quantities of interest are concentrations, to guarantee their non-negativity we let

$$
C_{j}=h\left(U_{j}\right)=C_{0, j} \exp \left(U_{j}\right)
$$

where $C_{0, j}$ is the value of the concentration at steady state, and reformulate the dynamical system for the metabolism in terms of the logarithms

$$
U_{j}=\log \frac{C_{j}}{C_{0, j}} .
$$

Due to the fact that the mass matrix $\mathrm{M}$ is diagonal, letting where $\Lambda(U)=\mathrm{M} \operatorname{diag}(h(U))$, the differential equation of the metabolism in terms of the dimensionless $U$ is of the form

$$
\begin{aligned}
\frac{d U}{d t} & =\Lambda(U)^{-1} F\left(h(U), \psi_{\text {ATPase }, \mathrm{n}}, \psi_{\text {ATPase }, \mathrm{a}}, q\right) \\
& =\Phi\left(U, \psi_{\text {ATPase }, \mathrm{n}}, \psi_{\text {ATPase }, \mathrm{a}}, q\right) .
\end{aligned}
$$

The solution of this differential equation is approximated using a numerical integrator based on the backward differentiation formulae (BDF) to properly handle the inherent stiffness of the system. Given the discrete time instances

$$
t_{j}=j \Delta t, \quad j=0,1, . . J_{\max }, \quad J_{\max }=\frac{T}{\Delta t}-1,
$$

and introducing the notation

$$
\begin{aligned}
U_{j} & =U\left(t_{j}\right), \\
\Phi_{j} & =\Phi\left(U_{j}, \psi_{\text {ATPase }, \mathrm{n}}\left(t_{j}\right), \psi_{\text {ATPase }, \mathrm{a}}\left(t_{j}\right), q\left(t_{j}\right)\right) .
\end{aligned}
$$


a BDF time integrator of order $r$ computes $U_{j}$ from the value of $U$ at the previous $r$ time nodes, $U_{j-\ell}, 1 \leq \ell \leq r$ and some scalars $\alpha_{j}$ and $\beta$ by solving an equation of the form

$$
\sum_{\ell=0}^{r} \alpha_{\ell} U_{j-\ell}=\Delta_{t} \beta \Phi_{j},
$$

with a few steps of the Newton method. We denote the $r$-step BDF-based integrator symbolically as $\mathcal{B}_{r}$, so that

$$
U_{j}=\mathcal{B}_{r}\left(\Phi_{j}, U_{j-1}\right)
$$

\subsection{Updating the electrophysiology}

The evaluation of $\Phi_{j}$ requires the values of the ATP dephosphorylation fluxes in neuron and astrocyte, which, in turn, are obtained by integrating numerically the electrophysiology model over the time interval $(j \Delta t,(j+1) \Delta t)$. Due to the smaller time constant of the electrophysiology process, the time step used for the numerical time integration is significantly smaller than for the metabolism. Therefore, to distinguish between the two time discretizations, we denote the time step for the metabolism by $\Delta t$, and the finer time step for the electrophysiology model by $\Delta \tau$. Moreover, since the electrophysiology system is independent of the time when is updated at the metabolic time scale, it can be computed on the interval $[0, \Delta t]$. For solving the electrophysiology model in our computed examples, we use the stiff time integrator ode15s in Matlab, with adaptive $\Delta \tau$. If $\tau=\left[\tau_{m}\right]_{m=0, \ldots, M_{j}}$ is the interpolation grid of the electrophysiology in $(0, \Delta t)$, at each $\tau_{m}$ we calculate the values of $J_{\text {pump,Na }}\left(\tau_{m}\right), J_{\text {glia, } \mathrm{K}^{+}}\left(\tau_{m}\right)$, and $I_{\mathrm{Na}^{+}, \text {act }}\left(\tau_{m}\right)$, and plug them into (23) and (24) to obtain the value of $\psi_{\text {ATPase }}\left(\tau_{m}\right)$. Since we are interested in the energetic needs for the electrophysiology between two successive updates of the metabolism, we can average $\psi_{\text {ATPase }}$ over the interval $(0, \Delta t)$, by a suitable quadrature rule. On the other hand, since the propagation of the electrophysiology model requires knowledge of the phosphorylation state, we propose the following predictor-corrector iteration scheme: in the first iteration, iwe assume the values of the phosphorylation rates $\mathrm{n}$ the electrophysiology to be the same at all $\tau_{m}$ and equal those computed at the previous metabolic time step, while in the second iteration we update their values using a linear interpolant between the previous values and the updated ones. The organization of the different computational steps is summarized below in the form of an algorithm. For each $j=0,1, \ldots, j_{\max }$, we replacing the time interval $[0, \Delta t]$ by the discrete time grid $\tau_{0}, \tau_{1}, \ldots, \tau_{m_{j}}$.

Introduce the notation

$$
X^{j, m}=X\left(j \Delta t, \tau_{m}\right)
$$

and

$$
\tau=\left[\begin{array}{c}
\tau_{0} \\
\tau_{1} \\
\vdots \\
\tau_{\mathrm{M}_{\mathrm{j}}}
\end{array}\right], \quad \mathbf{u}^{j, \tau}=\left[\begin{array}{c}
u^{j, 0} \\
u^{j, 1} \\
\vdots \\
u^{j, M_{j}}
\end{array}\right], \quad \mathbf{u}^{j}=\mathbf{u}^{j, 0}, \mathbf{u}^{j, M_{j}}=u^{j+1,0}
$$




\section{Algorithm 1 Multiscale Metabolic Update}

1. Given: the metabolite concentrations $\left\{U_{0}, U_{-1}, \ldots U_{-r+1}\right\}$ at the previous $r$ time instances

2. For $j=0, \ldots, J_{\max }$

Calculate $p_{n}^{j}$ and $p_{a}^{j}$ from (6).

Set $p_{n}^{j+1}=p_{n}^{j}, \quad p_{a}^{j+1}=p_{a}^{j}$

\section{Predictor Step:}

Define

$f_{n}(\tau)=p_{n}^{j}\left(1-\frac{\tau}{\Delta t}\right)+p_{n}^{j+1} \frac{\tau}{\Delta t}$

$f_{a}(\tau)=p_{a}^{j}\left(1-\frac{\tau}{\Delta t}\right)+p_{a}^{j+1} \frac{\tau}{\Delta t}$

Step 1: Compute $\mathbf{u}^{j+1, \tau}$ from $f_{n}(\tau), f_{a}(\tau)$ and $\mathbf{u}^{j}$ using a time integrator based on BDF;

Approximate $f_{n}(\tau)$ and $f_{a}(\tau)$ in $\tau$ by computing

$$
\begin{aligned}
& p_{\mathrm{n}}^{j, m}=\left(1-\frac{\tau_{m}}{\Delta t}\right) p_{\mathrm{n}}^{j, 0}+\frac{\tau_{m}}{\Delta t} p_{\mathrm{n}}^{j, M_{j}} \\
& p_{\mathrm{a}}^{j, m}=\left(1-\frac{\tau_{m}}{\Delta t}\right) p_{\mathrm{a}}^{j, 0}+\frac{\tau_{m}}{\Delta t} p_{\mathrm{a}}^{j, M_{j}}
\end{aligned}
$$

Evaluate $J_{\text {pump }, \mathrm{Na}^{+}}^{j, m}, J_{\mathrm{glia}, \mathrm{K}^{+}}^{j, m}$ and $I_{\text {act,Na }}^{j, m}$ at the fine scale from (18), (19) and (22).

Evaluate the ATPase fluxes in the fine scale from (23) and (24).

Update the ATPase fluxes in the coarse scale model:

$$
\begin{aligned}
& \psi_{\text {ATPase }, \mathrm{n}}((j+1) \Delta t)=\frac{1}{2 \Delta t} \sum_{m=0}^{M_{j}-1} \psi_{\text {ATPase }, \mathrm{n}}^{j, m}\left(\tau_{m+1}-\tau_{m-1}\right) \\
& \psi_{\text {ATPase } \mathrm{a}}((j+1) \Delta t)=\frac{1}{2 \Delta t} \sum_{m=0}^{M_{j}-1} \psi_{\text {ATPase } \mathrm{a}}^{j, m}\left(\tau_{m+1}-\tau_{m-1}\right)
\end{aligned}
$$

Step 2: Compute $U_{j+1}$ by integrating numerically $\Phi_{j+1}$ from (29) with a time integrator based on $\mathrm{BDF}$

$$
U_{j+1}=\mathcal{B}_{r}\left(\Phi_{j+1}, U_{j}\right) .
$$

Corrector Step: Update $p_{n}^{j+1}$ and $p_{a}^{j+1}$ via (6) using the most recent value of $U_{j+1}$

Repeat from Predictor Step

End $j$

Numerical evidence suggests that it suffices to repeat the Predictor-Corrector sequence twice. Note that while in the algorithm above the ATPase fluxes are computed by a midpoint quadrature rule, as we did in the numerical simulation presented in the following section, higher order quadrature rules can also be used.

\section{Results}

\subsection{Model calibration}

The computational predictive model of the integrated electrophysiology and metabolism depends on a number of parameters that need to be tuned properly to obtain meaningful results. This section is devoted to the initial calibration of the model. 


\subsubsection{Electrophysiology model}

It is well-known, and confirmed both using in vitro experiments and computationally, that the potassium concentration in the ECS has a major effect on neuron excitability. Consequently, the firing pattern of the electrophysiology model depends strongly on the four parameters that control directly the extracellular potassium level: $k_{\infty}, \varepsilon_{\mathrm{ECS}}$, $G_{\text {glia }}$, and $\rho$. In [17], the authors investigate the phase transitions between different firing patters with a reduced model, identifying regions in the parameter space that correspond either to seizure-like high-frequency firing bursts, followed by quiescent afterhyperpolarization firing gaps, as well as regions of steady state low frequency firing. Since our goal is to induce the firing mechanism indirectly by controlling the input functions $\xi$ and $q$ rather than setting the potassium level, we first find the value of the parameters so that the electrophysiology model, uncoupled from the metabolic model, can sustain a steady state of approximately $4 \mathrm{~Hz}$ background firing. The parameter values, listed in Table 9, are somewhat in agreement with the bifurcation diagrams in [17].

Following [7], in our electrophysiology model we neglect the calcium dynamics, and moreover we modify the partition of the potassium cleaning power between neuron and glia. In agreement with recent literature suggesting much higher values for the potassium cleaning in the neuron, if $T_{p}=\mathrm{G}_{\text {glia }}+\rho$ is the total potassium cleaning power in the two compartments, we assume that $60 \%$ is due to glia and the remaining $40 \%$ to neuron:

$$
\mathrm{G}_{\mathrm{glia}}=0.6 T_{p}, \rho=0.4 T_{p} .
$$

The value of $T_{p}=34.55 \mathrm{mM} / \mathrm{s}$ is computed from the values for $\mathrm{G}_{\mathrm{glia}}$ and $\rho$ presented in [17], while setting the potassium bath concentration $k_{\infty}$ to $6.3 \mathrm{mM}$ produces a $4 \mathrm{~Hz}$ background firing.

\begin{tabular}{|l|l|l|l|}
\hline Name & Symbol & Value & Units \\
\hline Potassium bath concentration & $k_{\infty}$ & 6.3 & $\mathrm{mM}$ \\
Diffusion coefficient & $\varepsilon$ & 9.33 & $\mathrm{~s}^{-1}$ \\
Glial uptake strength & $G_{\text {glia }}$ & 20.75 & $\mathrm{mM} / \mathrm{s}$ \\
Neuronal pump strength & $\rho$ & 13.83 & $\mathrm{mM} / \mathrm{s}$ \\
\hline
\end{tabular}

Table 9: Parameter values in the electrophysiology model corresponding to a $4 \mathrm{~Hz}$ background firing rate.

In [17] the neuron to extracellular volume compartment ratio $\beta$ is assumed to be 7 , while in the metabolic model [14], a significantly lower value $\beta=0.4 / 0.3=1.33$ was used. The former figure suggests that the volumes of neuron and astrocyte are lumped.

\subsubsection{Frequency calibration}

The electrophysiology model was tested independently of the metabolism in a variety of different conditions, by changing the activation function $\xi$ and observing the frequencies at which the system settled. These results are summarized in the plot shown in Figure 2 (left), where, not surprisingly, the increase of the conductance of sodium and potassium controlled through the function $\xi$ corresponds to an increase of the baseline frequency.

\subsubsection{OGI calibration}

The value $s$ of the parameter weighing the energetic is connected with the sodium leak, sodium-potassium pump and potassium cleaning, and that of the household energy $H_{1}$ in (23) are chosen so as to obtain values of the Oxygen Glucose Index (OGI), both at resting state and during neuronal sustained activity, in agreement with those reported in the literature, OGI is defined [37,39] as the ratio between the flux of oxygen and the flux of glucose from the 
blood compartment to the extracellular space:

$$
\mathrm{OGI}=\frac{J_{\mathrm{O}_{2}}}{J_{\mathrm{Glc}}}
$$

where $J_{\mathrm{O}_{2}}$ and $J_{\mathrm{Glc}}$ are calculated using equation (2). More precisely, we consider the OGI in two awake resting states, at tfiring frequencies of $8 \mathrm{~Hz}$ and $12 \mathrm{~Hz}$, which define the most common frequency band in human EEG $[10,28]$, and in a $90 \mathrm{~Hz}$ sustained neuronal activation for a simulation period of 3 minutes. The resting frequency of $8 \mathrm{~Hz}$ corresponds to an activation factor value of $\xi=0.06$, and the $12 \mathrm{~Hz}$ resting frequency to $\xi=0.15$, while to obtain a sustained $90 \mathrm{~Hz}$ frequency the activation factor was raised to $\xi=2.5$. In both cases we varied the household energy and the parameter $s$; in the middle and right panels of Figure 2 we show the corresponding values of OGI. The color maps represent the OGI levels for the resting states, while the red lines identify the OGI levels for the sustained activity. These experiments have been performed on the metabolic model, using as an input the energetic cost of the sodium potassium pump and glial potassium cleaning obtained from the electrophysiology model for each of the corresponding frequencies $(8 \mathrm{~Hz}, 12 \mathrm{~Hz}, 90 \mathrm{~Hz})$. During all the experiments, the household energy for the astrocyte was kept as: $H_{2}=0.833 H_{1}$. According to the literature $[33,37,40,39,36]$ we expect OGI values between 5 and 5.5 for resting states and between 4 and 4.5 for sustained neuronal activation. Owing to the results in Figure 2, in the rest of the paper we set $s=0.15$ and $H_{1}=4.3 \mathrm{mM} / \mathrm{min}$.


Figure 2: Frequency calibration (left panel): frequency sustained by the electrophysiology model as a function of the activation function $\xi$. OGI analysis (middle and right panels): cross scales parameter $s$ versus household energy $H_{1}$. The color coding in the plots identifies equi-OGI regions as the cross scaling parameter ranges from 0.15 to 2 and the household energy $H_{1}$ ranges from 3 to 4.5 , in the case of the sustained frequency of $8 \mathrm{~Hz}$ (left panel) and $12 \mathrm{~Hz}$ (right panel). In each panel the red curves indicate the sustained OGI levels at a firing frequency of $90 \mathrm{~Hz}$.

\subsection{Simulations}

\subsubsection{Protocol 1: consecutive activations}

In this computed example we simulate two consecutive neuronal activations, each lasting 3 minutes, with a recovery time in between them. During activation we set $\xi=2.5$ to sustain a firing rate frequency of $90 \mathrm{~Hz}$, while the system features a resting frequency of around $8 \mathrm{~Hz}$ elsewhere $(\xi=0.06)$. The hemodynamic response to the neuronal activation is to increase the blood flow hence the amount of oxygen and glucose available to the neuron. The blood flow increase follows the onset of the sustained neuronal activity with a delay of 2 seconds, and starts to decrease 5 seconds after the end of the activation. We model the blood response as a piecewise linear function, $q(t)=A(t) q_{0}$, where $A(t)$ is described in Table 11. In the first set of computer experiments, covering a time span of 30 minutes, the two activations start at $t_{i, 1}=2 \mathrm{~min}$ and $t_{i, 2}=15 \mathrm{~min}$, respectively, with a recovery interval of 10 minutes in between. The blood flow increases by about $30 \%$ over an interval of $r_{i}=10 \mathrm{~s}$ and decreases over an interval of $r_{f}=20 \mathrm{~s}$, while remaining continuous in time.

The first panel of Figure 3 shows the action potential and the estimated firing frequency in the 30 minutes of the first set of computer simulations. It is noticeable in the first panel that during sustained activation, the amplitude 
of the action potential decreases, as suggested in the literature [15]. At the beginning of each activation episode, there is a sharp increase in the firing frequency which reaches a peak of $107 \mathrm{~Hz}$ before stabilizing in few seconds at a steady frequency around $90 \mathrm{~Hz}$. At the end of the activations, the firing frequency returns to the baseline value of around $8 \mathrm{~Hz}$ following a short firing gap, known as slow after hyperpolarization (sAHP). Since its discovery in 1975, there has been a lot of interest in the sAHP, see, e.g., [23],[32],[38]. Our computed examples indicate that concurrently with the sAHP, which lasts approximately 12 seconds, the extracellular potassium concentration drops below its resting state value, as shown in the second panel. The last panel shows the time course of the intracellular concentration of the sodium and calcium ions: the intracellular sodium concentration drops from $17.6 \mathrm{mM}$ back to its normal value of $11.6 \mathrm{mM}$ in about 12 seconds, while the extracellular potassium increases from $6.29 \mathrm{mM}$ to $6.38 \mathrm{mM}$. Figures 4-5 show that the behavior of metabolites and electrophysiology during the second activation the same as during the first because the interval in between is long enough for the metabolites to return to their resting state values.

\begin{tabular}{|c|c|c|c|c|c|}
\hline Time & {$\left[t_{0}, t_{i}+d_{i}\right)$} & {$\left[t_{i}+d_{i}, t_{i}+d_{i}+r_{i}\right)$} & {$\left[t_{i}+d_{i}+r_{i}, t_{f}+d_{f}\right)$} & {$\left[t_{f}+d_{f}, t_{f}+d_{f}+r_{f}\right)$} & {$\left[t_{f}+d_{f}+r_{f}, T\right)$} \\
\hline$A(t)$ & 1 & $1+\delta \frac{t-t_{i}-d_{i}}{r_{i}}$ & $1+\delta$ & $e^{-\alpha\left(t-t_{f}-d_{f}\right)} a+b$ & 1 \\
\hline
\end{tabular}

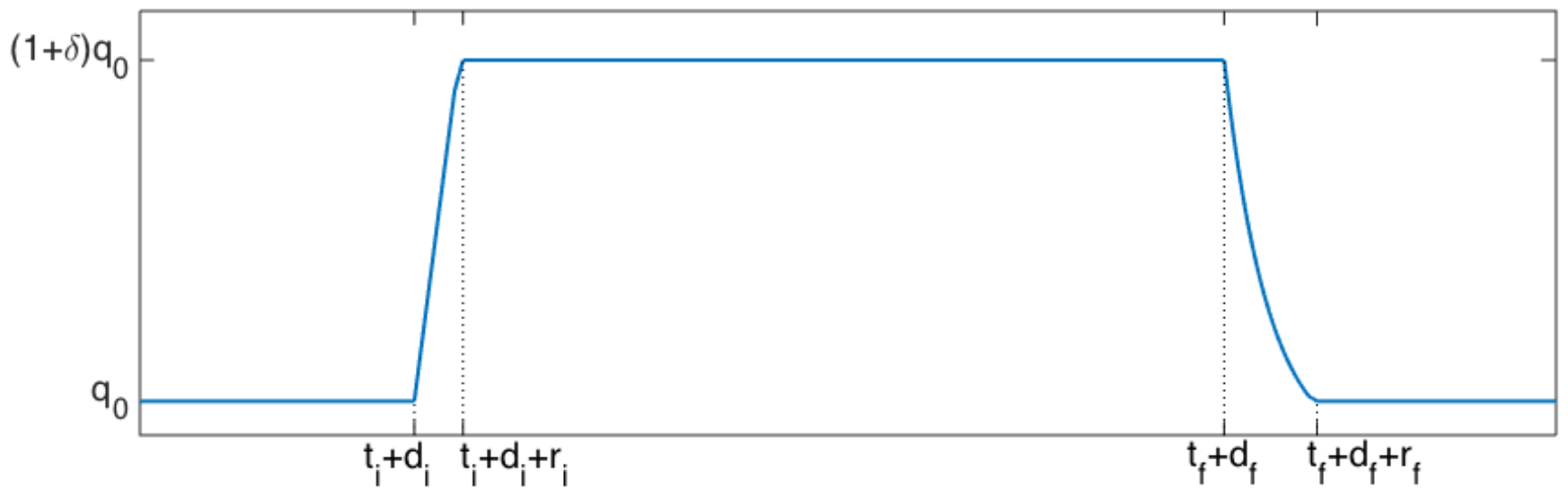

Table 10: Blood Flow regulation during sustained neuronal activation episode. The blood flow activation function used to simulate one activation epoch. In the top row of the table are indicated the time intervals where the function is defined as described in the corresponding cell in the bottom row. The resulting blood flow time course is plotted in the graph underneath the table. Here $q_{0}$ is the blood flow baseline, $\delta$ denotes the blood flow increment over the baseline, $t_{i}$ and $t_{f}$ are the initial and final times of the activation event, $d_{i}$ and $d_{f}$ delays in the blood flow response to activation start and stop, $r_{i}$ and $r_{f}$ are the ramping times (in seconds) response to the start and end of the stimulus. The values of the parameters $\alpha, a$ and $b$ are chosen so that the blood flow is continuous throughout the experiment. In the first set of computed examples, $\delta=0.3, d_{i}=2 \mathrm{~s}, d_{f}=5 \mathrm{~s}, r_{i}=10 \mathrm{~s}, r_{f}=20 \mathrm{~s}, \alpha=0.1$, $a=0.35$, and $b=0.95$. 

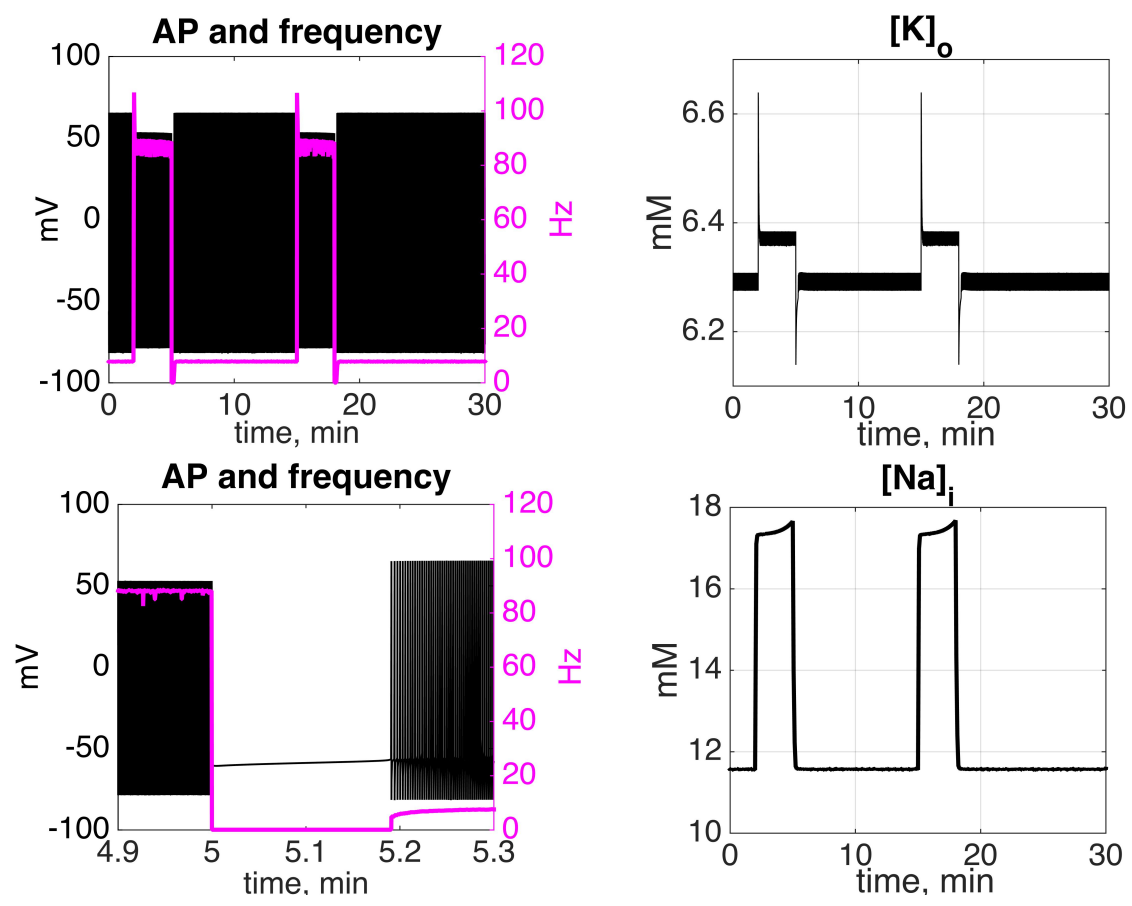

Figure 3: Consecutive activations with a 10 minutes interval between). Upper row. Left: action potential (black) and corresponding firing frequency (magenta). Right: time course of extracellular potassium concentration. Bottom row: Detail of the action potential and frequency around the time $t=5 \mathrm{~min}$ when the first activation is stopped. Right: time course of intracellular sodium concentration.

During sustained activation, our computed results show an increase in glucose consumption, whose concentration decreases, accompanied by an increase in lactate concentration: see: Figure 5, top row, left and middle panels. More specifically, in neuron and extracellular space the decrease in glucose concentration from baseline is approximately $60 \%$, and even higher in astrocyte, where it is over $85 \%$. The lactate concentration, on the other hand, exhibits a $65 \%$ increase over baseline in neuron, astrocyte and extracellular space, while in the blood, the increase is only $36 \%$. Oxygen consumption during sustained activation varies widely in the different compartments: the decrease in oxygen concentration during the two activity episodes reaches $90 \%$ in neuron, $73 \%$ in extracellular space and $50 \%$ in astrocyte. In the blood compartment there is a $4.3 \%$ increase in oxygen level during activation, followed by a marked dip at its end. The second row of Figure 4 shows, from left to right, the fluxes of the different metabolites from blood to the extracellular space and from extracellular space to neuron and astrocyte, respectively. At resting state, the flux of oxygen from blood to extracellular space is around $1.5 \mathrm{mM} / \mathrm{min}$, within the 1.4 to $1.7 \mathrm{mM} / \mathrm{min}$ range documented in the literature $[46,33,1]$. Notice that during the sustained activation the increase in the flux of glucose of approximately $38 \%$, and that of oxygen of $15 \%$ are very close to the values observed experimentally by Madsen et al. [36].

Some comments are in order about the time needed for the metabolites to return to their initial states. In top left panels of Figure 4 and Figure 5 we can see that by the beginning of the second activation, at $t=15$ min, concentrations have recovered to $98 \%$ of their initial values control value in neuron and extracellular space. Lactate, on the other hand, seems slower to recover: at $\mathrm{t}=15 \mathrm{~min}$, its concentration is $95 \%$ of its resting state value in neuron, astrocyte and extracellular space. In the blood compartment, both glucose and oxygen concentrations quickly return to their baseline values, while 10 minutes after the end of the first activation period, lactate concentration is $96 \%$ of its resting value.

In Figure 6 we plot the reaction fluxes, the time course of the cerebral metabolic oxygen rate of glucose, and the time course of the OGI. The latter is showing a dynamics in agreement with the literature.

We investigated how rapidly the metabolites return to their steady state values after the activation ceases by per- 
forming similar simulations varying only the time between the activations. We summarized our findings in Figure 7. In each example, the glucose concentration at the beginning of the second activation was compared to its baseline value, while we compared the peak concentration of lactate at the end of the second activation to that at the end of the first activation. Figure 7 shows that when the interval between the two activations is 2 minutes, glucose concentration in neuron at the start of the second activation is only $67 \%$ of its resting state value, and glucose concentration in astrocyte even lower, $54 \%$ of its resting state value, whereas when the interval increases to 20 minutes, all metabolite concentrations have returned to their baseline values at the beginning of the second activation.


Figure 4: Consecutive sustained activations with a 10 minute interval between. Time course of metabolite concentrations in the blood (top row): glucose (left), lactate (middle), and oxygen (right). Metabolic fluxes between compartments (bottom row): oxygen (black), lactate (red), and glucose (blue) between blood and extracellular space (left), between extracellular space and neuron (middle), and between extracellular space and astrocyte (right). 

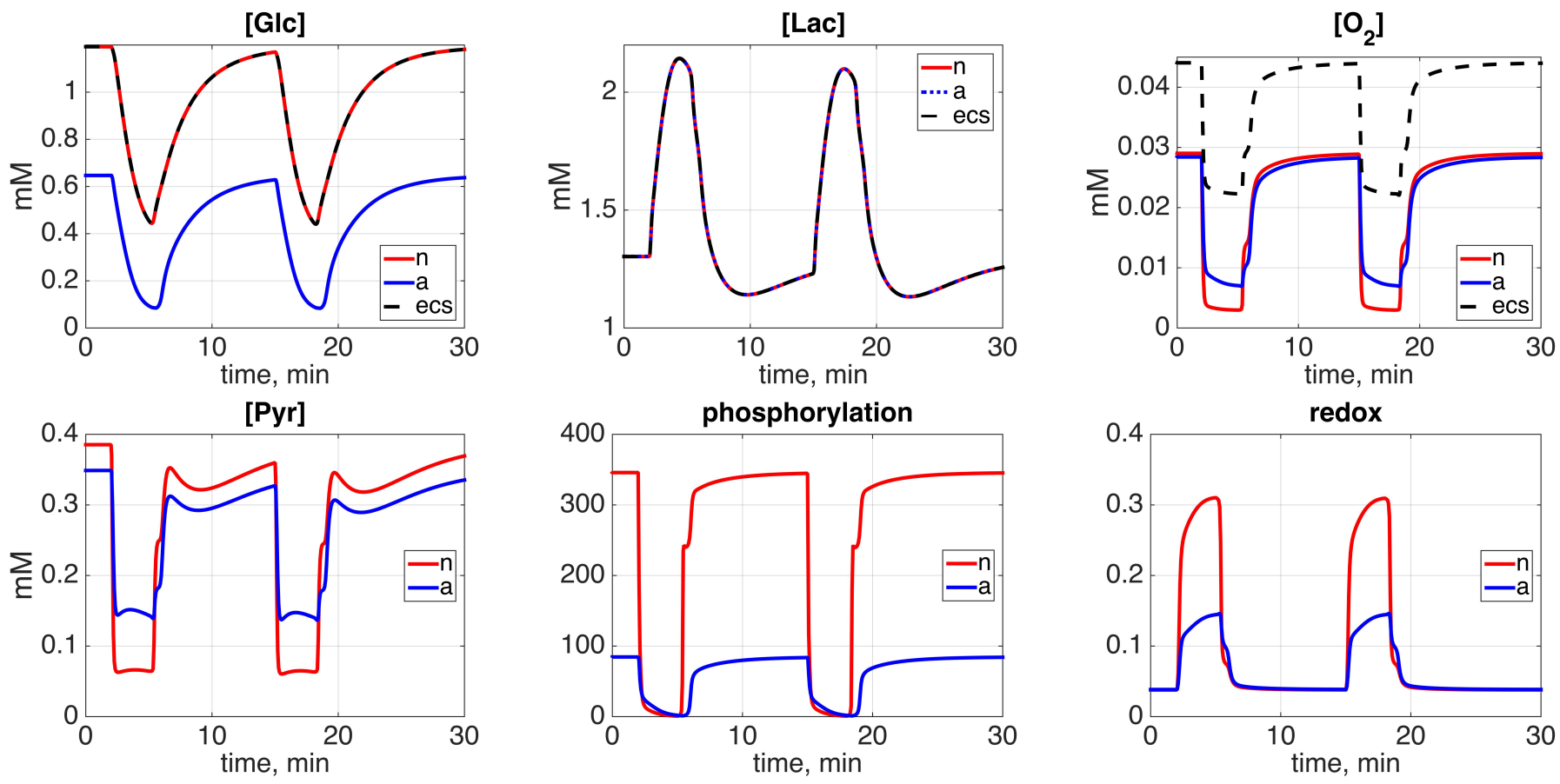

Figure 5: Consecutive sustained activations with a 10 minute interval between. Time course of metabolite concentrations in the neuron (in red), astrocyte (blue) and extracellular space (black): glucose (top left), lactate (top middle), oxygen (top right), and pyruvate (bottom left). Time course of phosphorylation (bottom middle) and redox (bottom right) states. 

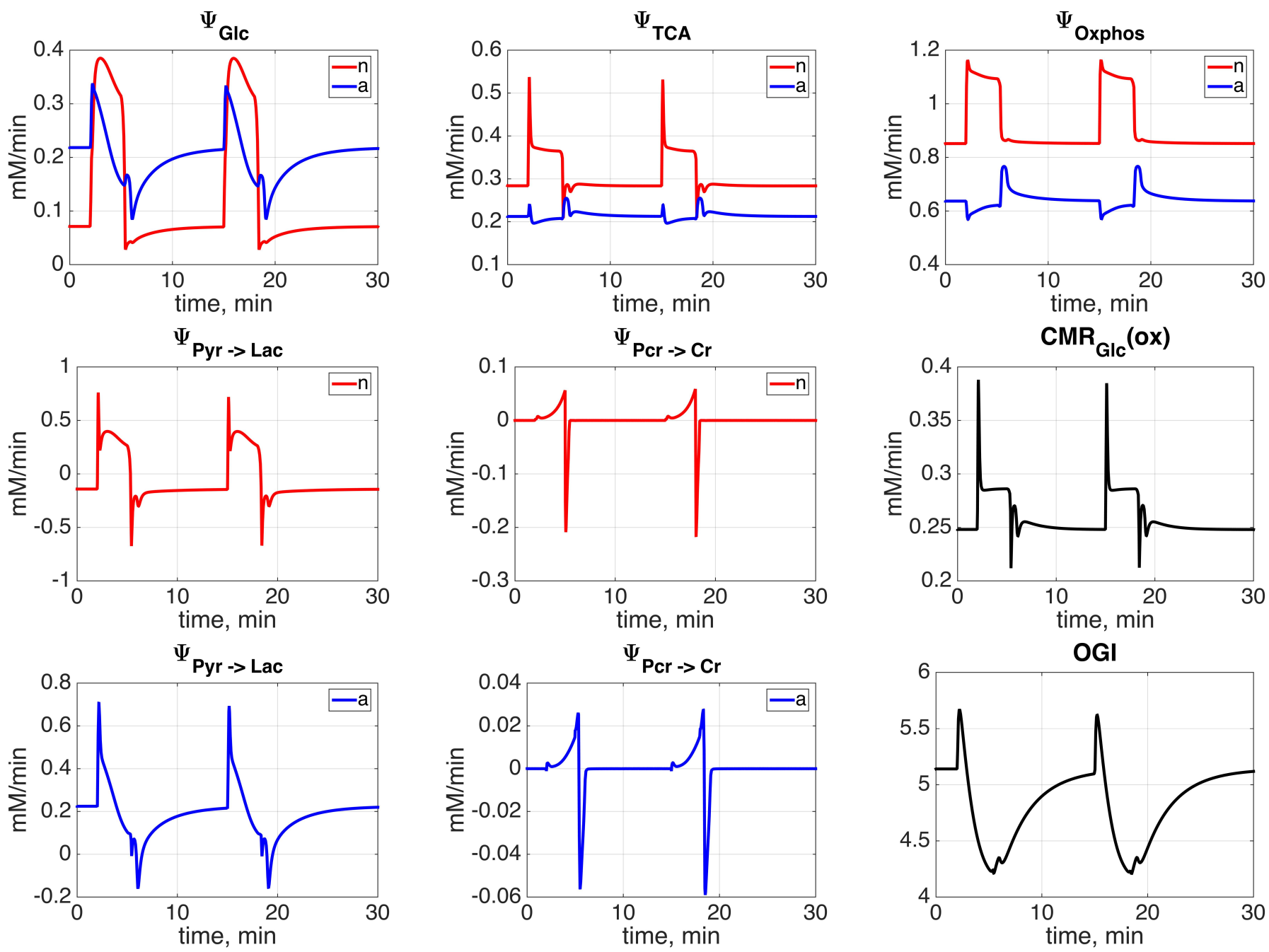

Figure 6: Consecutive sustained activations with a 10 minute interval between. Reaction fluxes. Top row: glycolysis (left), TCA (middle) and oxidative phosphorylation (right) in neuron (red) and astrocyte (blue). Middle row: lactate dehydrogenase balance flux $\left(\Psi_{L D H 1}-\Psi_{L D H 2}\right.$, left), and creatine phosphorylation balance flux $\left(\Psi_{P C r}-\right.$ $\Psi_{C r}$, middle) in the neuron; time course of the cerebral metabolic oxygen rate of glucose (right). Bottom row: lactate dehydrogenase balance flux $\left(\Psi_{L D H 1}-\Psi_{L D H 2}\right.$, left), and creatine phosphorylation balance flux $\left(\Psi_{P C r}-\Psi_{C r}\right.$, middle) in astrocyte; time course of the OGI index (right).
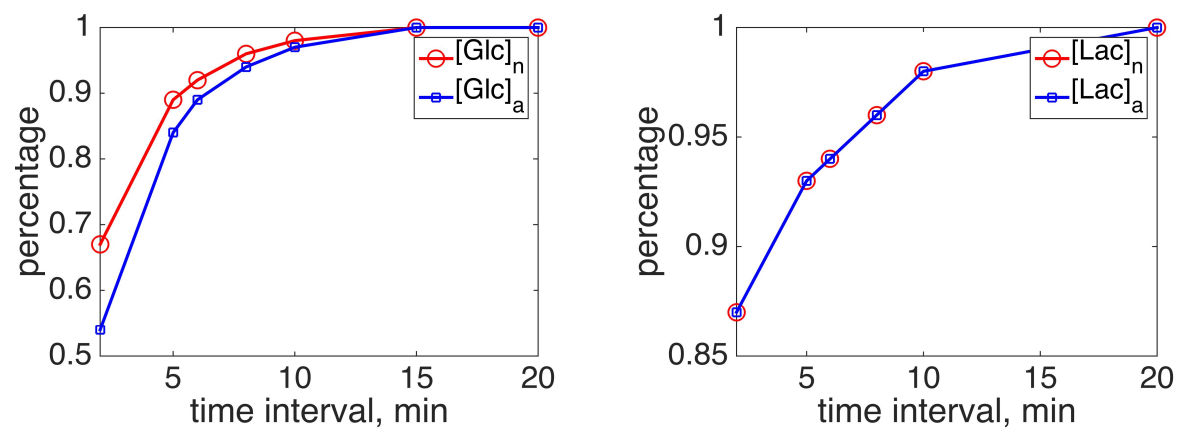

Figure 7: Return to initial conditions vs length of time interval between consecutive activations Relative recovery of glucose (left) and lactate (right) concentration in neuron (red) and astrocyte (blue) at the start of the second activation as a function of the interval between consecutive activations. 


\subsubsection{Protocol 2: Transient ischemia from awake resting steady state}

In this computer simulation we study how changes in blood flow affect the electrophysiology by considering an ischemia protocol where the blood flow is reduced by $90 \%$ for a period of 1.5 minutes. The details of the piecewise linear function effectuating the blood flow reduction are presented in Table 11, together with its graph.

\begin{tabular}{|c|c|c|c|c|c|}
\hline Time & {$\left[t_{0}, t_{1}\right)$} & {$\left[t_{1}, t_{1}+r_{1}\right)$} & {$\left[t_{1}+r_{1}, t_{2}\right)$} & {$\left[t_{2}, t_{2}+r_{2}\right)$} & {$\left[t_{2}+r_{2}, T\right)$} \\
\hline$A(t)$ & 1 & $1-\delta \frac{t-t_{1}}{r_{1}}$ & $1-\delta$ & $1-\delta\left(1-\frac{t-t_{2}}{r_{2}}\right)$ & 1 \\
\hline
\end{tabular}

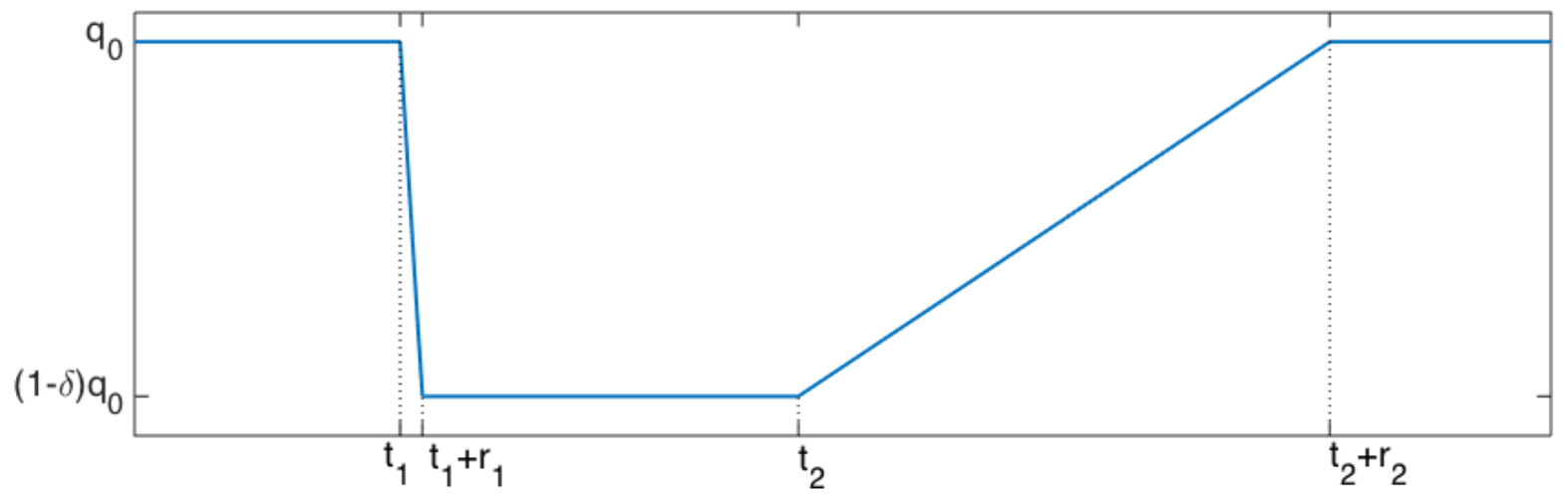

Table 11: Blood flow regulation during transient ischemia. The blood flow activation function used to simulate one ischemic episode. In the top row of the table are indicated the time intervals where the function is defined as described in the corresponding cell in the bottom row. The resulting blood flow time course is plotted in the graph underneath the table. Here $q_{0}$ is the blood flow baseline, $\delta$ denotes the blood flow reduction from its baseline value, $t_{1}$ and $t_{2}$ are the initial and final moment of the ischemic episode, $r_{1}$ and $r_{2}$ are the ramping times (in seconds) for the blood flow to decrease to its minimum and return to its resting value, respectively. In this computed example we set $\delta=0.9, t_{1}=2 \mathrm{~min}, t_{2}=3.5 \mathrm{~min}, r_{2}=5 \mathrm{~s}$, and $r_{2}=120 \mathrm{~s}$.

The ischemic episode, which lasts $T_{d}=1.5$ minutes, starts $t_{1}=2$ minutes after the beginning of the simulation, and the blood flow decreases to $10 \%$ of its value at awake resting state within $r_{1}=5$ seconds from the onset of the ischemia, and at the end of the episode gradually returns to the starting value over a period of $r_{2}=2$ minutes. The different role that neuron and astrocyte have in sustaining neuronal firing suggest that the reduction of blood flow during ischemia will affect the two cells and their functions in different ways. More specifically, we expect the decreased glial metabolic activity during the ischemic episode to trigger a rise in the potassium concentration in ECS, in turn increasing the excitability of the neuron. On the other hand, reduced oxygen availability in neuron makes the pump action non sustainable, hence slowing down or even stopping the firing. Indeed, Figure 8 shows a slight elevation of the extracellular potassium level. Moreover, the increased levels of lactate in neuron and astrocyte indicate that because of the much decreased oxygen availability, the cells try to match ATP requirement by relying mostly on anaerobic metabolism, whose lower efficiency is unable to maintain ATP turnover at the same level as before the ischemia, thus causing a drop in activity. 

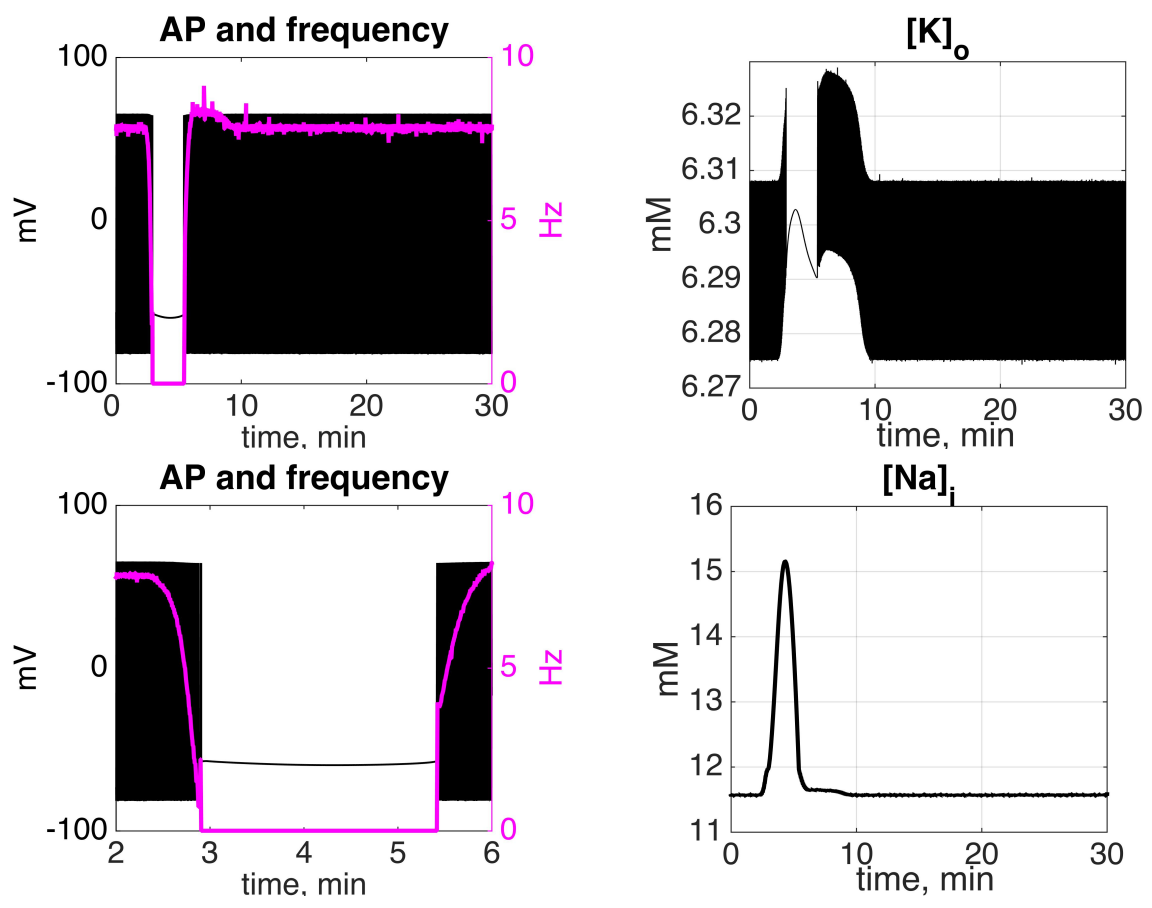

Figure 8: Transient ischemia: electrophysiology. Upper row: Action potential (black) and corresponding firing frequency (magenta) in the left panel, and time course of extracellular potassium concentration in the right one. Bottom row: Detail of the action potential around the the time of the ischemic event in the left panel, and the time course of intracellular sodium concentration in the right one.

Figure 9 shows a drop in oxygen and glucose concentration concomitantly with the reduction in blood flow. The glucose concentration decay is more marked in astrocyte, where it is close to $90 \%$ of its baseline value, than in neuron and extracellular space, where it is a little over $60 \%$. At the same time, in blood compartment the decrease is a slightly above $50 \%$. The oxygen concentration decreases to almost zero in blood, neuron and astrocyte, and is only slightly higher, at $15 \%$ of its baseline value, in extracellular space,. There is sharp increase in lactate concentration in neuron, astrocyte and extracellular space, where it is more than double its baseline value, as well as in blood, where it is a little less than double.

The first panel of the bottom row of Figure 10 shows a more marked decay in the concentration of pyruvate in neuron, where it decreases by approximatively $95 \%$ of its baseline value, than in the astrocyte, where the decay is around $90 \%$ of baseline value. The middle panel shows that after 90 seconds of ischemia, the phosphorylation states in both neuron and astrocyte get almost entirely depleted; concurrently, the surge in redox in neuron is approximately double than in astrocyte, as illustrated in the right panel in the same row.

The time courses of the fluxes of the reaction included in the metabolism portion of our model are shown in Figure 11. We remark that during the ischemic episode the rate of glycolysis raises sharply, while the TCA cycle and oxidative phosphorylation slow down significantly, to rebound sharply above baseline values once the blood flow returns to baseline value. The first two panels in the last row of Figure 11 show that the inhibition of oxidative metabolism is accompanied by a surge in anaerobic metabolism, as indicated by the increased production of lactate from pyruvate and a high rate of phosphocreatine kinase to meet the demand for ATP. The last panel shows a marked decrease, about 50\%, of OGI during the ischemic episode, and the rather long time, approximately 20 minutes, before it returns to baseline levels. 

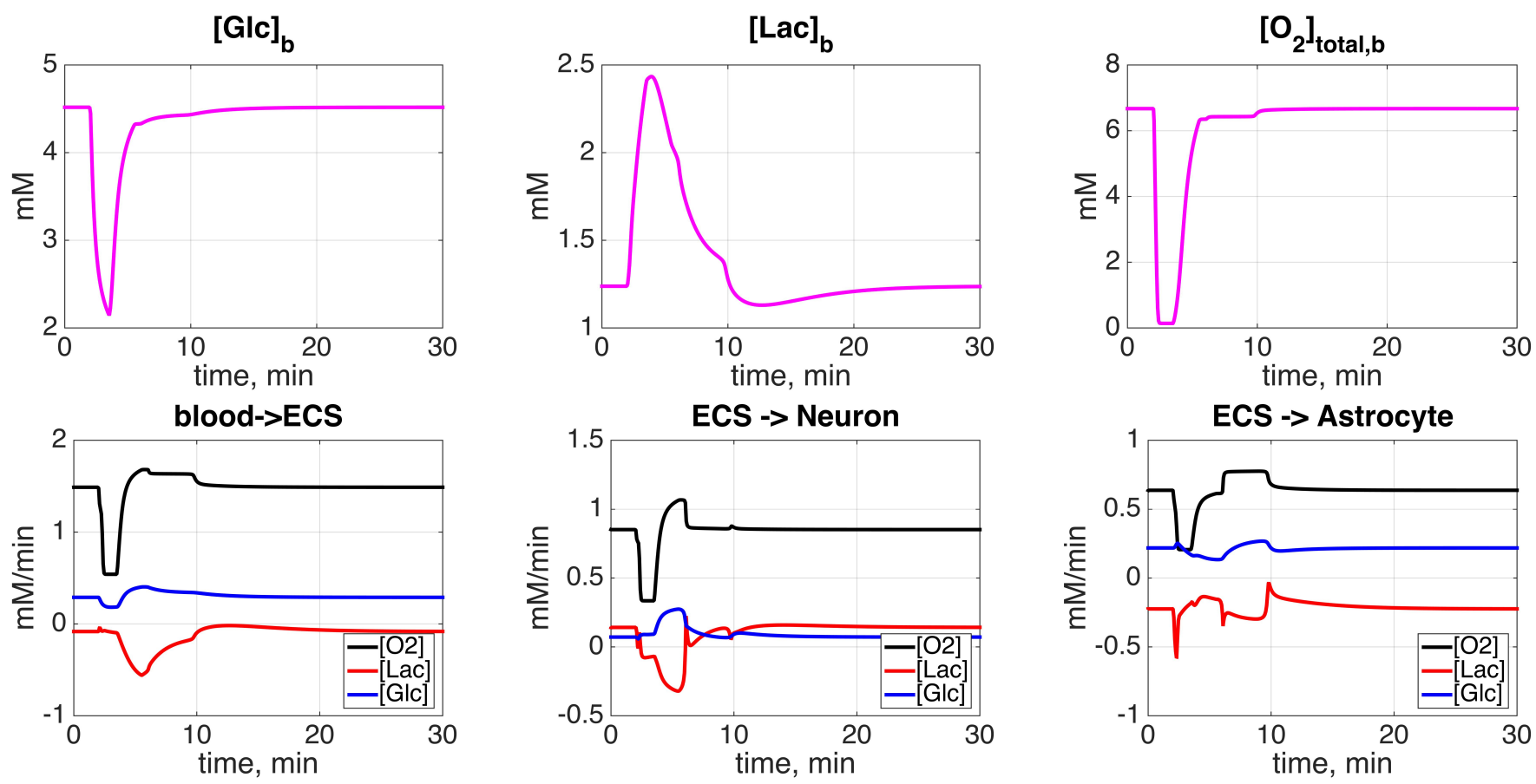

Figure 9: Transient ischemia: blood concentrations and transport rates. Time course of metabolite concentrations in blood (top row): glucose (left), lactate (middle), and oxygen (right). Transport rates fluxes between compartments (bottom row): oxygen (in black), lactate (ired), and glucose (blue) between blood and extracellular space (left), between extracellular space and neuron (middle), and between extracellular space and astrocyte (right).
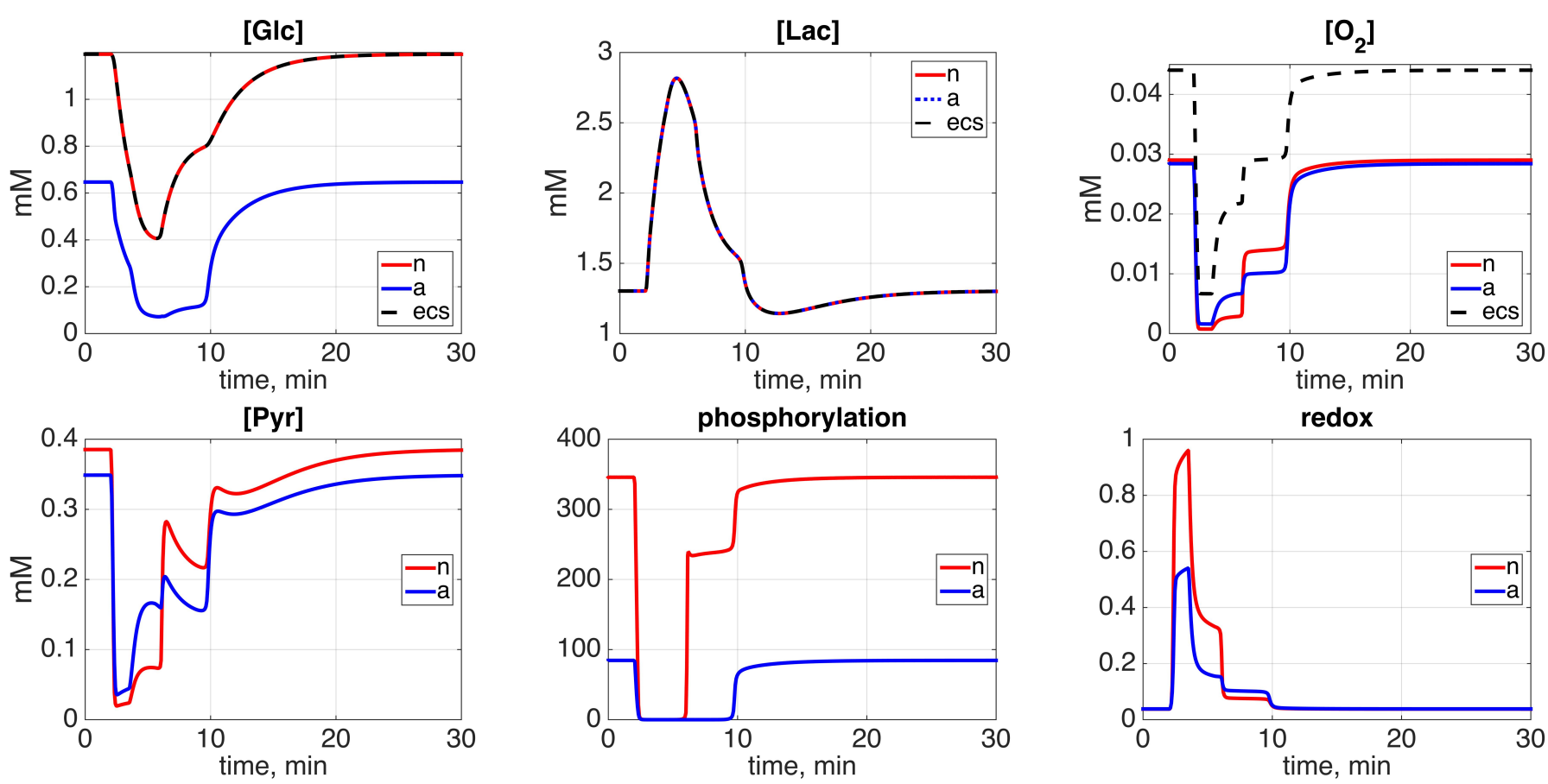

Figure 10: Transient ischemia: metabolite concentrations. Time course of metabolite concentrations in neuron (red), astrocyte (blue) and extracellular space (black): glucose (top left), lactate (top middle), oxygen (top right), and pyruvate (in neuron and astrocyte, bottom left). Time course of phosphorylation (bottom middle) and redox (bottom right) states in neuron and astrocyte. 

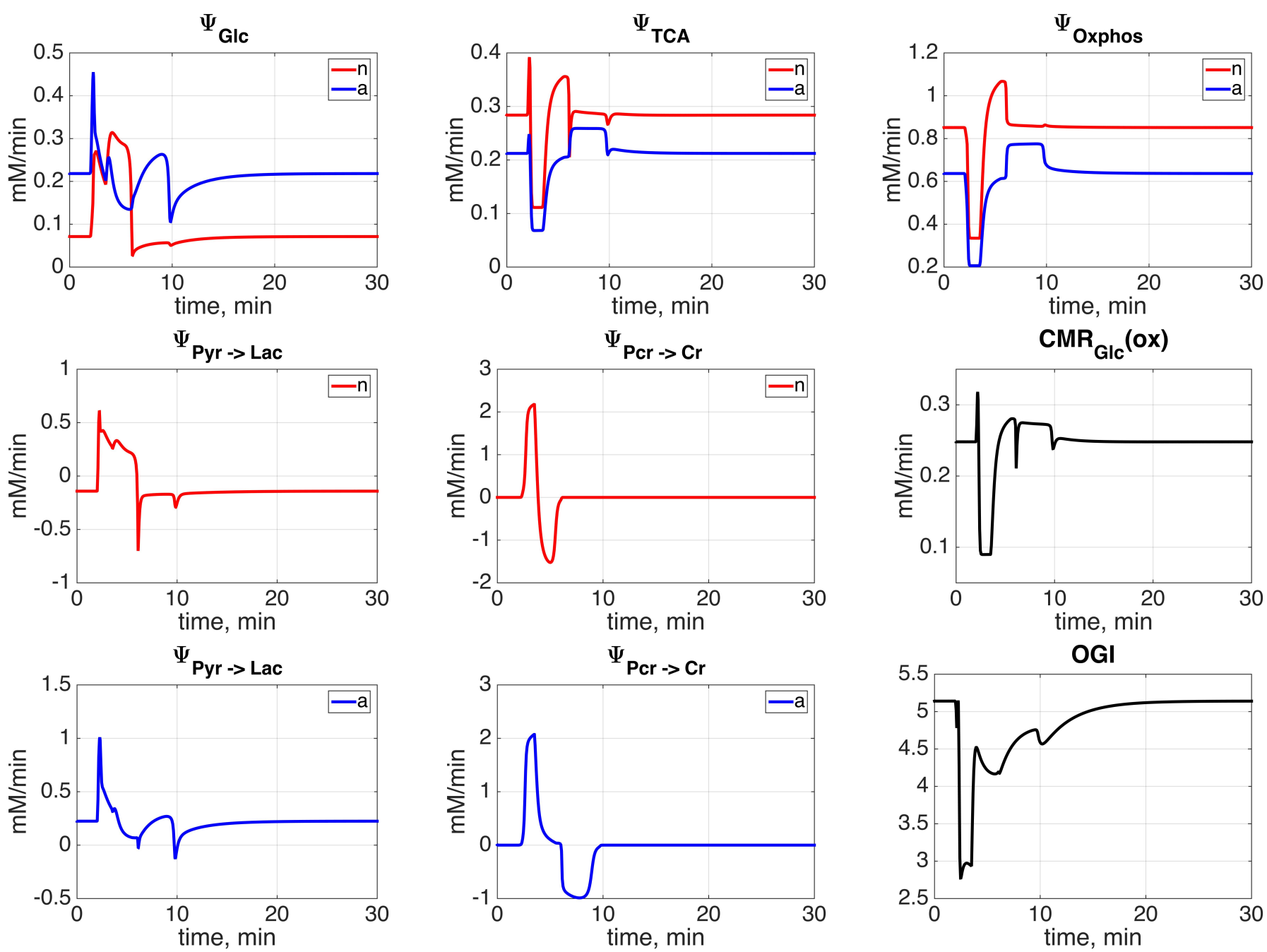

Figure 11: Transient ischemia: reaction fluxes. Reaction fluxes. Top row: glycolysis (left), TCA cycle (middle) and oxidative phosphorylation (right) in neuron (red) and astrocyte (blue). Middle row: lactate dehydrogenase balance flux, with positive direction indicating lactate production (left), creatine phosphorylation balance flux (middle) in neuron and oxidative cerebral metabolic rate of glucose (right). Bottom row: lactate dehydrogenase balance flux, with positive direction indicating lactate production (left), creatine phosphorylation balance flux (middle) in astrocyte and time course of the OGI index (right).

\subsubsection{Protocol 3: Transient ischemia followed by activation}

This protocol is a combination of the first two, simulating a transient ischemic episode from resting awake steady state, followed by an activation period. The ischemic episode starts $t_{1}=2$ minutes into the simulation and ends at $t_{2}=3.5$ minutes, followed, after 10 minutes, by a period of sustained activation starting at $t_{i}=13.5$ minutes and ending at $t_{f}=16.5$ minutes. The blood flow dynamics is a combination of the ones described in the previous protocols, and the values of the parameters $\alpha, a$ and $b$ are as in Protocol 1. Moreover, as in Protocol 1, we set $\xi=2.5$ during activation, to attain a sustained firing frequency of $90 \mathrm{~Hz}$.

Figure 12 shows that, as in Protocol 2, the firing frequency decreases to 0 during the ischemic event and starts recovering back to its baseline value once the ischemic event is stopped, eventually returning to its baseline frequency of $8 \mathrm{~Hz}$ at time $t=5.5 \mathrm{~min}$. After 8 minutes of firing at baseline awake resting state frequency, at the onset of the neuronal activation, the frequency first shows a sharp peak of $107 \mathrm{~Hz}$, then settles at the active steady frequency of $90 \mathrm{~Hz}$. As in Protocol 1, at the end of the activation period our model predicts a short firing gap, corresponding to 
the slow after hyperpolarization effect observed experimentally. The right panel of Figure 12 shows a higher concentration of sodium and potassium during the sustained activation than in the 90 seconds of the transient ischemic event.


Figure 12: Transient ischemia followed by activation. Left: action potential (black) and corresponding firing frequency (magenta). Middle: time course of the extracellular potassium concentration. Right: time course of intracellular sodium concentration.

Figures 13 and 14 show that at the end of the ischemic episode, the concentrations of most metabolites return to their awake resting state values, and although the time to full recovery is different for each metabolite and compartment, at the start of the activation event, most of them are back to more than $80 \%$ of their awake resting value. We remark that the production of lactate increases both during ischemia and sustained activation.

The bottom row of Figure 13 shows the time course of the transport rate of glucose and oxygen from blood to the extracellular space (left) and from extracellular space to neuron and astrocyte (middle). Notice that after the end of ischemia and before the activation, the flux of glucose from blood to extracellular space is $104.5 \%$ of its baseline value, while the flux of oxygen is at its baseline value, which leads to an OGI which reaches $96 \%$ of its resting state value (Figure 15). The efflux of lactate into blood increases significantly during the ischemic episode and sustained activation, with both neuron and astrocyte producing rather than consuming it. 

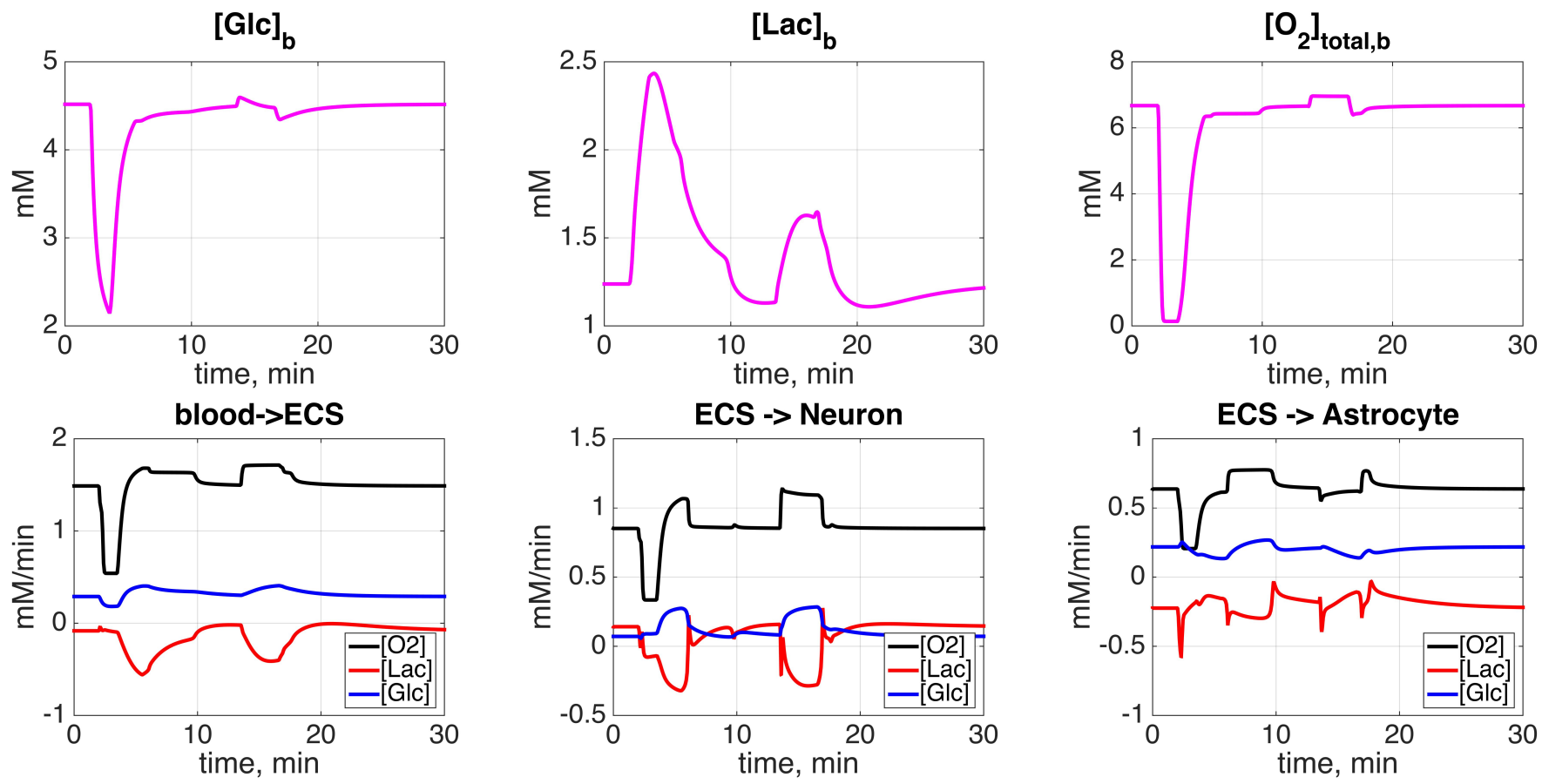

Figure 13: Transient ischemia followed by activation Time course of metabolite concentrations in blood (top row): glucose (left), lactate (middle), and oxygen (right). Transport rates between compartments (bottom row): oxygen (in black), lactate (in red), and glucose (in blue) between blood and extracellular space (left), between extracellular space and neuron (middle), and between extracellular space and astrocyte (right).
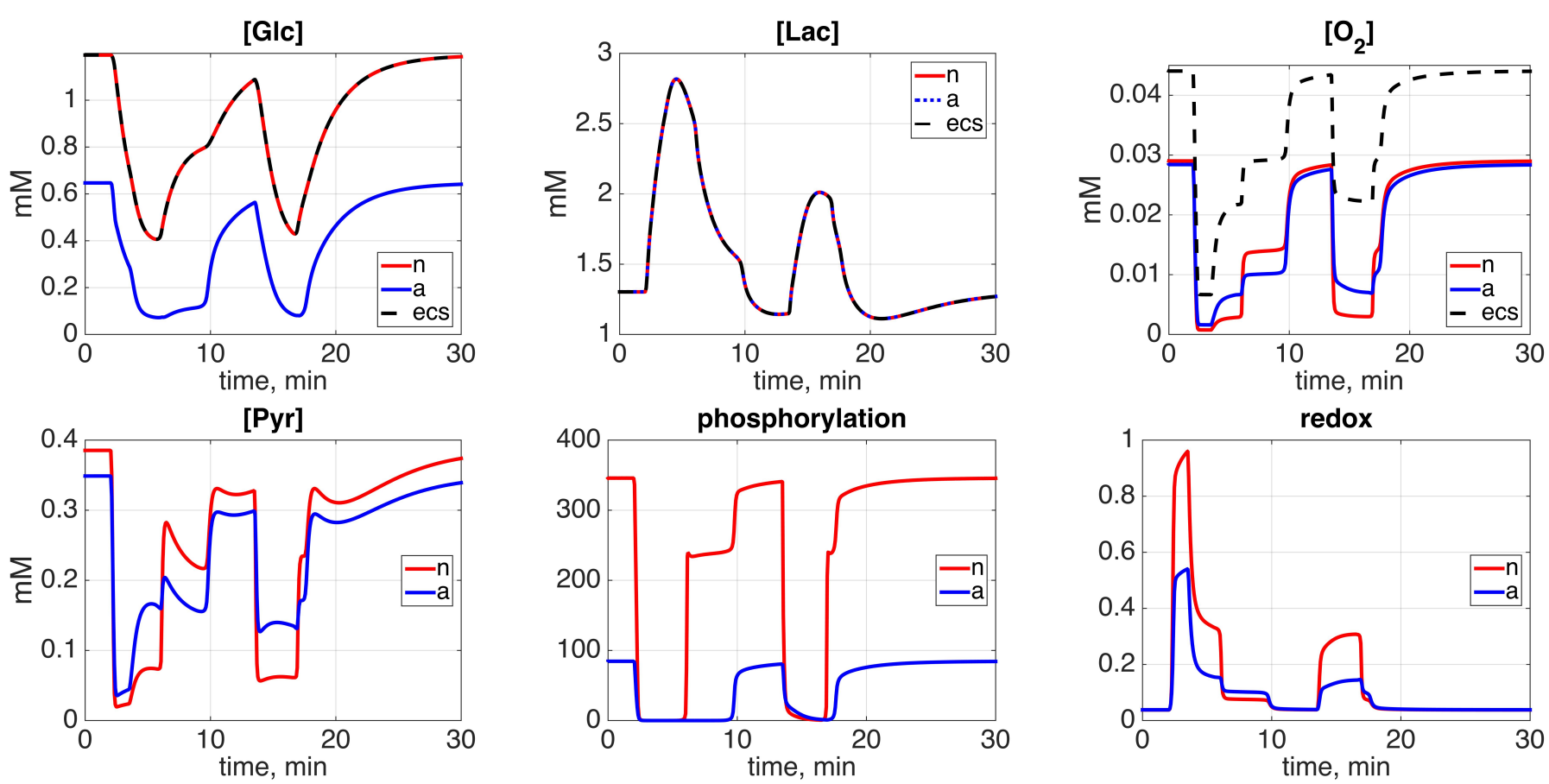

Figure 14: Transient ischemia followed by activation. Time course of metabolite concentrations in neuron (in red), astrocyte (in blue) and extracellular space (in black): glucose (top left), lactate (top middle), oxygen (top right), and pyruvate (bottom left). Time course of phosphorylation (bottom middle) and redox (bottom right) states in neuron and astrocyte. 

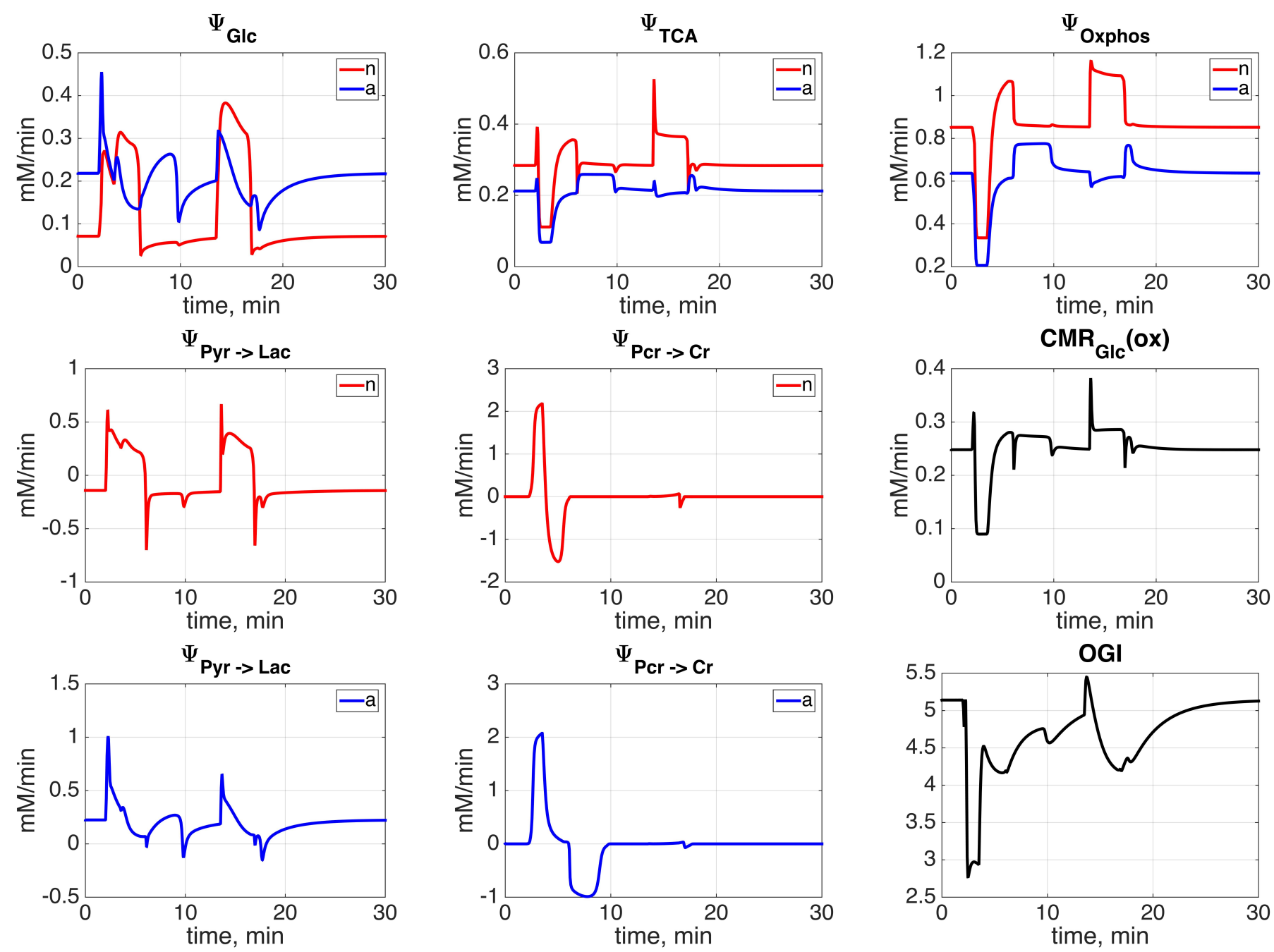

Figure 15: Transient ischemia followed by activation. Reaction fluxes. Top row: glycolysis (left), TCA cycle (middle) and oxidative phosphorylation (right) in neuron (red) and astrocyte (blue). Middle row: lactate dehydrogenase balance flux $\Psi_{L D H 1}-\Psi_{L D H 2}$ in neuron (left), creatine phosphorylation balance flux $\Psi_{P C r}-\Psi_{C r}$ in neuron (middle); cerebral metabolic oxygen rate of glucose (right). Bottom row: lactate dehydrogenase balance flux $\Psi_{L D H 1}-\Psi_{L D H 2}$ in astrocyte (left); creatine phosphorylation balance flux $\Psi_{P C r}-\Psi_{C r}$ in astrocyte (middle); OGI index (right).

\subsubsection{Partitionig of signaling energy and dependency of firing on blood flow}

The variation over time of the ATP turnover going towards signaling in the three different protocols, displayed in Figure 16, shows that the majority of the energy expenses due signaling occurs in neuron during the excitatory events. The ischemic episode causes a rapid drop in signaling energy in both neuron and astrocyte, but while in the latter the return to baseline level coincides with with normalization of the blood flow, in astrocyte this requires several more minutes, suggesting that the signaling functions of astrocyte may be affected more severely by ischemia. 

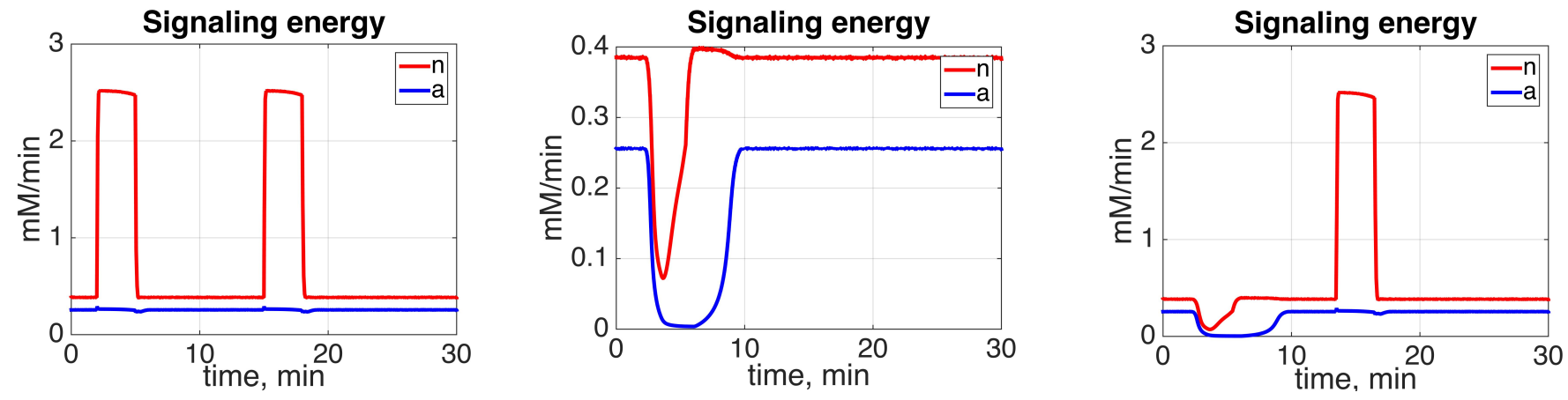

Figure 16: ATP turnover from the signaling acticity: Protocol1 (left): two consecutive activation events; Protocol 2 (middle): a transient ischemia episode; Protocol 3 (right) a transient ischema followed by an activation event.

Another question of interest is how long, given a steady blood flow, the neuron-astrocyte unit can sustain firing at a certain frequency. The left panel in Figure 17 shows that, at baseline blood flow $q_{0}$, the system can sustain firing at a frequency higher than $70 \mathrm{~Hz}$ for less than 10 minutes, but has no problem sustaining firing at $60 \mathrm{~Hz}$ for as long as one hours. A $30 \%$ higher blood flow, on the other hand, makes it possible sustained firing for at least one hour at $80 \mathrm{~Hz}$, whereas with a reduction of the blood flow to $30 \%$ of its baseline value the firing frequency that the system can sustain for at least one hour drops below $20 \mathrm{~Hz}$. The right panel shows the dependency of the OGI on the firing frequency with different values of the blood flow. The figure indicates a clear phase transition from predominantly aerobic metabolism (OGI over 5) to anaerobic metabolism (OGI around 4), the critical firing frequency depending on the blood flow. For lower blood flow, the insufficient oxygen supply limit is reached at lower level of energetic demand.
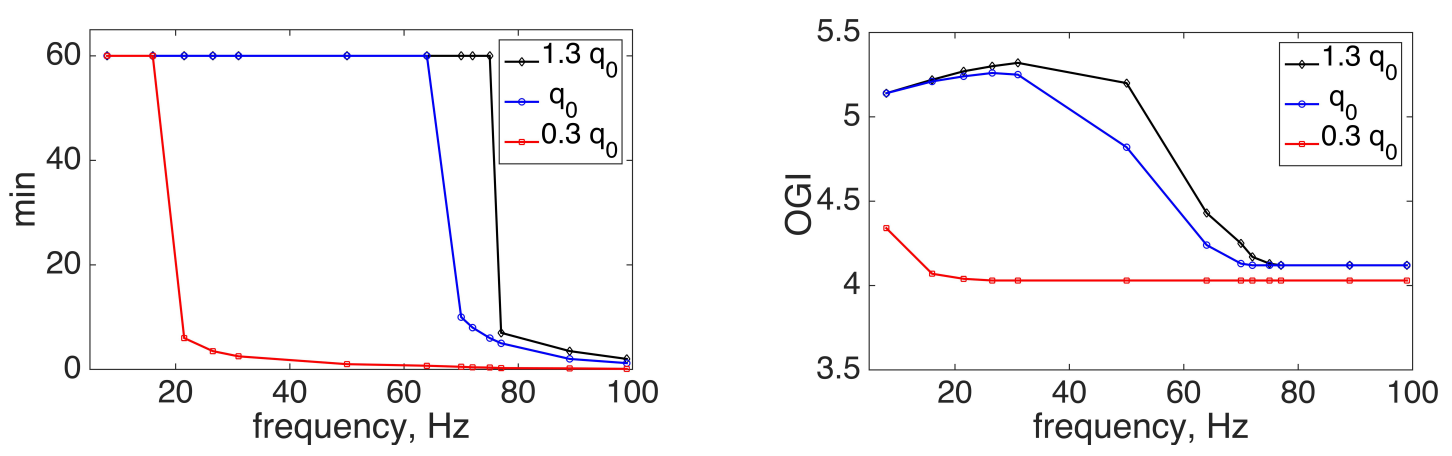

Figure 17: Left: maximum time interval during which the system can sustain firing at a given frequency as a function of the cerebral blood flow. Right: Dependency of the OGI on the firing frequency and the blood flow. The phase transition from high to low OGI marks the switch from aerobic to anaerobic metabolism.

\section{Discussion}

A remarkable feature of our model is its ability to describe the electrophysiological and metabolic states at different activation levels by specifying changes in the activation parameter $\xi$ regulating current conductances. The values of $\xi$ associated with the different states of neuronal activation were chosen after a careful calibration study to make sure that the resulting firing frequency were in agreement with experimental findings reported in the literature.

Volume compensation in coupling electrophysiology and metabolism: The coupling between electrophysiology and metabolism in our model accounts for the energetic cost of neurotransmission in the mass action equations for ATP in neuron and astrocyte and assumes that all the energy released by the ATP dephosphorylation goes towards 
household chores needed for general cell functions and neurotransmission, described in terms of ion currents. The differences in the volumes of the relevant compartments in the electrophysiology and metabolism are bridged with the introduction of a coupling parameters $s$, whose value depends on the brain region of interested but is independent of the neuronal activity level. The criterion guiding the selection of the value of $s$ was that the model predictions should be in agreement with results reported in the neurophysiology literature, hence factoring in the energetic demand for housekeeping tasks. In response to an increase in the value of the activation parameter $\xi$, the higher $\mathrm{Na}^{+}$ and $\mathrm{K}^{+}$leak conductances induce a rise in the firing frequency: the higher energy needed to sustain the $\mathrm{Na}^{+}-\mathrm{K}^{+}$ pump activity and the potassium clearing drives a change in the metabolism of both neuron and astrocyte together with an increase of the cerebral blood flow to sustain such changes.

Volume of the metabolic compartments: In this paper we keep the compartment volumes fixed in all protocols except during ischemia, as the focus is on the energetic coupling between electrophysiology and metabolism. During an ischemic episode, the ATP production may be insufficient to maintain the functioning of the Na-K pump, yielding an increase in the intracellular sodium concentration and a concomitant swelling of the cell. To account for this we increase by $50 \%$ the volume fraction of neuron and astrocyte, reducing the volume fraction of ECS accordingly. The changes in the model predictions are by and large fairly insensitive to the volume changes during ischemia.

Blood Flow: Variations in blood flow in response to neuronal activity levels play an important role, and must be taken into account. In this paper we consider the blood flow as an input function, described by a simple, phenomenological, piecewise model: a $30 \%$ flow increase is induced during the neuronal activation with a ramping period both at the beginning and the end of the activation to account for the delayed response of the cardiovascular system. Although the model presented in this paper is quite simple, it captures the essential behavior of the blood dynamics. An extension of this model including the hemodynamics response through neurovascular coupling is currently under development, and will be the subject of future work.

Metabolite transport from blood to tissue: Our model assumes that metabolites are transported from blood to cell via ECS, despite the fact that capillaries are covered by the end feet of the astrocytes, which would suggest a different transport pattern. The argument that astrocytes have preferred access to glucose has been used in the literature to justify the controversial Astrocyte-Neuron Lactate Shuttle (ANLS) Hypothesis [8]. Our modeling choice is based on the observation that only the glucose that enters a cell (neuron or astrocyte) and is phosphorylized by hexokinase (HK) to Glucose-6-phosphate (G6P) cannot return to ECS, while in the absence of HK, on the other hand, glucose entering a cell can return to ECS by GLUT transporters and within our simplified model, the traffic of non-phosphorylized glucose does not change the metabolism. On the other hand, in order for astrocyte to have primacy to glucose, their end feet should be rich on HK, a hypothesis which has been questioned in [35].

Oxygen transport and OGI: The mathematical expression of the oxygen transport flux from blood to tissue is non-linear modification of the classical Fick's law which assumes that the transport flux depends linearly on the free oxygen gradient. In numerical simulations (not shown), the classical Fick's model led to an increase rather than a decrease of the OGI during neuronal activation, in conflict with what reported in the literature [21]. A physiological argument supporting our non-linear model is the hypothesis that aquaporins may have a role as preferred gas channels, a topic that is under active investigation $[9,42]$.

Glutamine-glutamate cycling: In the current model, the glutamate-glutamine cycling between neuron and astrocyte is omitted, as the focus is on the energetic coupling of electrophysiology and metabolism rather than following the cycling of the neurotransmitters, whose addition would increase the complexity to the metabolism model. The energetic cost of the cycling has been previously addressed by the authors [12,13], and it is well established (see, e.g., [6]) that the main energetic cost is related to electrophysiology, not to transmitter cycling. The current modeling paradigm is chosen so that the emphasis remains on the former.

Assessment of household energy: In our model the household energy in the two cell types is higher for neuron than for astrocyte, in agreement with many literature contributions. This is the only energetic demand that has to be met at baseline; the additional energetic requirement going towards neuronal activation encompasses the additional aggregate costs, including glutamate recycling, sodium potassium pump and potassium cleaning. The estimates of the housekeeping cost in the two cell types used in our model were obtained through an extensive study and make 
the predictions of the model agree with experimental electrophysiological and metabolic measurements reported in the literature for different dynamic protocols.

Redox balance, potassium clearing and oxidative metabolism during activation: The first of our computed protocols looks at how a system initially in awake resting state, characterized by low frequency firing, undergoes two periods of 3 minutes of neuronal activation that raises the firing frequency to $90 \mathrm{~Hz}$, with a 10 minute interval at awake resting state in between. The sharp rise in firing frequency is accompanied by a rapid increase in ATPase going towards meeting energy demand for signaling in neuron but not in astrocyte. The surge in energetic demand in neuron and the concomitant increase in blood flow changes the concentrations of metabolites in blood, and the oversupply of glucose and oxygen initially is taken up by neuron. The rate of glycolysis in neuron increases during activation to return below the initial level in the interval between activations (see Figure 6), while in astrocyte the rate of glycolysis peaks briefly at the start of the activation then during the activation period falls below baseline level, where it return after a long recovery period. During activation, oxidative phosphorylation rate settles at a higher level than baseline in neuron, after a rapid initial overshoot, and at a lower level in astrocyte after a rapid undershoot at the beginning of the activation and significant, longer overshoot at the end of the activation, which is probably when astrocyte uses the available oxygen to meet the energetic demand of potassium clearing. At baseline condition, the neuron oxidizes lactate after converting it into pyruvate, but during activation pyruvate in converted into lactate to offset the rise of redox. In astrocyte, the prevailing direction of lactate dehydrogenase is towards lactate production except for a very short time after neuronal activation has ceased, concomitantly with a rise in oxidative phosphorylation rate. The sharp drop in both neuron and astrocyte of phosphorylation to near zero at the very start of activation and its remaining there until the end of activation interval and the decay in oxygen concentration in both cells indicate that all ATP produced by the system, aerobically and anaerobically, is used to meet the increased energy demand. The OGI plot shows an overshoot by over $10 \%$ at the start of the activation, followed by an almost $20 \%$ decay with a return to the resting value only by the time when the second activation starts. This is agreement with the observation about nonoxidative glucose consumption during activation in [21].

Time after activation to restore initial conditions: The model predictions highlight the key role of oxygen in meeting the energy demands of the system during activation and the metabolic response of astrocyte to the energetic cost of extracellular potassium clearing. An interval of approximately 10 minutes between period of sustained neuronal activation is needed for the system to return to the initial resting metabolic state. During activation the model allows the cerebral blood flow to increase by $30 \%$ of its baseline value: in computer simulations (not shown) where the blood flow was held constant the system was unable to sustain the neuronal activity for more than a few seconds, in agreement with the observation that in active areas of the brain there is an increase in blood flow.

Ischemia stops AP and affects astrocyte longer than neuron: The second simulation protocol illustrates that our model produces realistic predictions of how the system reacts to an episode of transient partial ischemia lasting 90 seconds and highlights how the effects of a decreased blood flow continue after the blood flow has returned to normal levels. The onset of ischemia is accompanied by an almost immediate drastic decrease of glucose concentration in blood and nearly complete depletion of oxygen, while the lactate concentration surges, indicating that once all available oxygen has been used up, the system tries to meet the energetic demands anaerobically. The production of ATP during the ischemia episode is not enough to sustain firing at $8 \mathrm{~Hz}$ frequency, hence firing stops for 90 seconds and restarts once the cerebral blood flow returns to normality, however for a brief period the frequency is above 8 Hz. Not surprisingly, the time of higher firing rate is characterized by elevated extracellular potassium concentration. The explanation for the latter comes from the time courses of phosphorylation, which stays near zero in astrocyte for some time after the end of the ischemic episode, suggesting that the astrocyte may not be able to carry our its potassium clearing function efficiently. During the period of the ischemia, the intracellular concentration of sodium increases because the ATP produced is not enough to keep the $\mathrm{Na}^{+}-\mathrm{K}^{+}$pumps active, to return to its baseline value once the blood flow is back to normal. The electrophysiology model adopted in this paper is a modification of the classical HH model that does not include the active sodium-potassium pump functions. The pump action under normal conditions at resting potential is accounted for by the concentration dependency of the reversal potentials (13) that in the classical model are assumed constants, leading to firing activity similar to the classical HH model. Hence, as the pump action stops due to the lack of ATP, the current model does not return to normal firing state as 
the HH model would do; rather, the sodium and potassium fluxes have a cancelling effect in the equation (8) of the transmembrane potential, and the normal firing restarts only after the pump functions are restored.

Elevated extracellular potassium after ischemic episode: The concentration of potassium in extracellular space increases in the post-ischemia period, and it requires several minutes before it returns to its baseline values, indicating that the ischemia impedes the potassium cleaning function of astrocyte for a prolonged period. The concentration of glucose in blood falls to less than $50 \%$ of its baseline value and that of oxygen drops all the way to zero while the lactate concentration doubles. This is an indication of the fact that to meet the energetic needs required for household functions with a much lower cerebral blood flow, the system runs the aerobic metabolism at capacity, with oxygen availability as limiting factor, while transforming a portion of the glucose into lactate to keep redox in a physiological range. While the duration of the ischemia is limited to 90 seconds, the changes induced in the metabolism of the system last10 times as long, whereas the disruption to the electrophysiological activity, which can be attributed to accumulation of potassium in extracellular space as a result of insufficient oxygen delivery to astrocyte, is of much shorter duration. Upon restoration of the cerebral blood flow the phosphorylation in neuron recovers to $2 / 3$ of the baseline value, while it takes the astrocyte longer to recover its baseline value, probably due to the need of cleaning the extracellular potassium accumulated during the ischemia.

Ischemia and activation affect differently redox, not phosphorylation: The third simulation protocol comprises a transient ischemic episode of the duration of 90 seconds with a blood flow $30 \%$ of its baseline, followed by an activation period of the same duration, starting 10 minutes after the end of the ischemia. At the end of each episode, glucose concentrations return to baseline values rapidly while lactate concentrations remain elevated for several more minutes, causing an acidification of the environment. As in protocols 2, phosphorylation decreases suddenly to near zero in both neuron and astrocyte, as expected. In neuron, phosphorylation bounces back to to about two thirds of its resting value a few minutes after ischemia ends, returning to baseline 6 minutes after the ischemia ends. In astrocyte, however, phosphorylation remains at zero twice as long, The changes in redox state, on the other hand, are more substantial during ischemia than during activation, with a behavior that is inversely proportional to the concentration of oxygen in blood. This observation confirms the fact that phosphorylation state is an indicator of the rate at which the energetic potential of the cells is used, while redox state tracks how the energy used by the cells is produced. The rate of oxidative phosphorylation, which decreases to near zero in both cells during ischemia, increases during activation in neurons while slightly dropping in astrocyte. The 20\% drop in OGI during activation suggests that a larger portion of glucose undergoes anaerobic transformation to lactate during activation than at resting state, thus highlighting the importance of redox balance.

Large increase in lactate concentration characterizes ischemic episode: The effects of ischemia and neuronal activation on the concentration of glucose are very similar, except that in the former it takes longer for the astrocyte to return to baseline concentration after the episode is over, whereas the rise in lactate concentration is both higher and longer lasting in response to a blood flow restriction. The height and duration of the lactate peaks correlate with the redox peaks: the ischemic episode is characterized by much higher redox and lactate concentration in neuron, while phosphorylation changes do not show much difference between ischemia and activation.

\section{Conclusions and future directions}

In this paper we introduce a predictive mathematical model that, relying on energy exchange, integrates a detailed electrophysiology model with detailed sodium-potassium dynamics model and a metabolic compartment model of a neuron-astrocyte complex. The model has been validated using literature values. From the methodological point of view, a novel component of the model is how the different time scales of electrophysiology and metabolism are integrated. A central contribution of this work is the setup of a double feedback mechanism between electrophysiology and metabolism, including the effect of extracellular potassium dynamics: the electrophysiology is regulated through the availability of ATP produced by the metabolism, and, conversely, the energy production is driven by the energetic needs of the electrophysiology. The overall driver of the system is an activation factor function that modulates the firing frequency of the neurons. In physiological terms, the activation represents a signal propagated 
from outside the region of interest. In particular, by tracking the extracellular potassium concentration in time, the model sheds some light on the role and the energetic cost of $\mathrm{K}^{+}$buffering. Another external control is provided by the blood flow, regulating the availability of the metabolites. The former control is used to generate activation states, while the latter allows a simulation of ischemic episodes.

The energetic costs attributed in our model to household chores, to signaling during period of neuronal activity, and to potassium glial cleaning, together comprise the link between the electrophysiology and the metabolism, giving rise to a system that can correctly predict the changes in substrates during, before and after a period of activation characterized by an increase in neuronal firing frequency as well as during, before and after an episode of transient ischemia. While both neuron and astrocyte shoulder the cost of neuronal activation and ischemia, the asymmetric roles of the different cells become evident in how they return to to initial conditions in the post-ischemia or postactivation periods. Our simulations demonstrate the key role of the extracellular potassium cleaning by the astrocyte in maintaining the system at a sustainable state: Increased extracellular potassium concentration during neuronal activation and - to a lesser extent - following an episode of transient ischemia have to be reset to baseline values by the potassium cleaning function of astrocytes, an operation that requires energy obtained by the process of breaking the energetic phosphate bond in ATP. Our numerical simulations show a longer depletion of the phosphorylation state in the astrocyte than in the neuron, highlighting the cost of such process. In addition, from our simulations we observe that a reduction of the cerebral blood flow to $30 \%$ of its baseline value for more than 90 seconds drives the system into a non sustainable state.

In the current model, the blood flow is controlled independently from the activation state. A more realistic model should involve a feedback mechanism between the metabolic functions, including the potassium dynamics, and the control of the blood flow. While some existing metabolic models include a module representing the blood flow giving rise to the BOLD-fMRI signal, a detailed description of the feedback is missing, and remains a future challenge.

Acknowledgements. This work was supported by the Bizkaia Talent and European Commission through COFUND under the grant CIPAS: Computational Inverse Problems Across Scales (AYD-000-278, 2015), by the Basque Government through the BERC 2014-2017 program, and by the Spanish Ministry of Economics and Competitiveness MINECO through the BCAM Severo Ochoa excellence accreditation SEV-2013-0323 and the Spanish "Plan Estatal de Investigación, Desarrollo e Innovación Orientada a los Retos de la Sociedad" under Grant BELEMET Brain ELEctro-METabolic modeling and numerical approximation (MTM2015-69992-R). The work of Daniela Calvetti was partly supported by Grant Number 246665 from the Simons Foundation, and the work of Erkki Somersalo was partly supported by NSF Grant DMS 1016183. Daniela Calvetti and Erkki Somersalo were partly supported by NIH, grant 1U01GM111251-01.

\section{References}

[1] J. Aanerud, P. Borghammer, M. M. Chakravarty, K. Vang, A. B. Rodell, K. Y. Jónsdottir, A. Møller, M. Ashkanian, M. S. Vafaee, P. Iversen, P. Johannsen, A. Gjedde, Brain energy metabolism and blood flow differences in healthy aging, J.Cereb. Blood Flow Metab. 32 (7) (2012) 1177-1187. doi:10.1038/jcbfm.2012.18.

[2] A. Aubert and R. Costalat. A model of the coupling between brain electrical activity, metabolism, and hemodynamics: application to the interpretation of functional neuroimaging. NeuroImage (2002) 17:1162-1181.

[3] A. Aubert, R. Costalat. Interaction between astrocytes and neurons studied using a mathematical model of compartmentalized energy metabolism. J. Cereb. Blood Flow Metab. 25 (2005)1476-1490.

[4] A. Aubert, R. Costalat, P. J. Magistretti, L. Pellerin. Brain lactate kinetics: modeling evidence for neuronal lactate uptake upon activation. Proc. Natl Acad. Sci. U.S.A. 102 (2005)16448-16453.

[5] A. Aubert, R. Costalat, R. Valabrègue. Modelling of the coupling between brain electrical activity and metabolism. Acta. Biotheor. 49 (2001) 301-326. 
[6] D. Attwell, S. B. Laughlin, An energy budget for signaling in the grey matter of the brain, J.Cereb. Blood Flow Metab. 21 (10) (2001) 1133-1145.

[7] E. Barreto, J. R. Cressman, Ion concentration dynamics as a mechanism for neuronal bursting, J. Biol. Physics 37 (3) (2011) 361-373. doi:10.1007/s10867-010-9212-6.

[8] M. Bélanger, I. Allaman, and P.J. Magistretti, P. J. Brain energy metabolism: focus on astrocyte-neuron metabolic cooperation. Cell metabolism, 14(6) (2011) 724-738.

[9] W.F. Boron, Sharpey-Schafer Lecture: Gas channels. Experimental Physiology, 95.12 (2010) 1107-1130.

[10] C. P. Brötzner, W. Klimesch, M. Doppelmayr, A. Zauner, H. H. Kerschbaum, Resting state alpha frequency is associated with menstrual cycle phase, estradiol and use of oral contraceptives, Brain Research 1577 (100) (2014) 36-44. doi:10.1016/j.brainres.2014.06.034.

[11] D. Calvetti, E. Somersalo. Large scale statistical parameter estimation in complex systems with an application to metabolic models. Multiscale Model. Simul. 5 (2006) 1333-1366.

[12] D. Calvetti, E Somersalo, Dynamic activation model for glutamatergic neurovascular unit. J. Theor. Biol. 274 (2011) 12-29.

[13] D. Calvetti, Y. Cheng, E. Somersalo, Uncertainty quantification in flux balance analysis of spatially lumped and distributed models of neuron-astrocyte metabolism, J. Math. Biol. 73 (6) (2016) 1823-1849. doi:10.1007/s00285-016-1011-7.

[14] D. Calvetti, Y. Cheng, E. Somersalo, A spatially distributed computational model of brain cellular metabolism, J.Theor. Biol. 376 (2015) 48-65. doi:http://dx.doi.org/10.1016/j.jtbi.2015.03.037.

[15] B. C. Carter, B. P. Bean, Sodium entry during action potentials of mammalian central neurons: incomplete inactivation and reduced metabolic efficiency in fast-spiking neurons, Neuron 64 (6) (2009) 898-909. doi:10.1016/j.neuron.2009.12.011.

[16] Cloutier, M., F. B. Bolger, J. P. Lowry, An integrative dynamic model of brain energy metabolism using in vivo neurochemical measurement. J. Comput. Neurosci. 27 (2009) 391- 414.

[17] J. R. Cressman, G. Ullah, J. Ziburkus, S. J. Schiff, E. Barreto, The influence of sodium and potassium dynamics on excitability, seizures, and the stability of persistent states: I. single neuron dynamics, J. Comput. Neuroscience 26 (2) (2009) 159-170. doi:10.1007/s10827-008-0132-4.

[18] G.A. Dienel, Fueling and imaging brain activation, ASN Neuro 4 (5) (2012) e00093. doi:10.1042/AN20120021.

[19] M, DiNuzzo, S. Mangia, B. Maraviglia, F. Giove, Changes in glucose uptake rather than lactate shuttle take center stage in subserving neuroenergetics: evidence from mathematical modeling. J. Cereb. Blood Flow Metab. 30 (2010) 586-602.

[20] F. Du, X.-H. Zhu, Y. Zhang, M. Friedman, N. Zhang, K. Uğurbil, W. Chen, Tightly coupled brain activity and cerebral atp metabolic rate, Proc. Nat. Acad. Sci. U.S.A. 105 (17) (2008) 6409-6414. doi:10.1073/pnas.0710766105.

[21] P. T. Fox, M. E. Raichle, M. A. Mintun, C. Dence, Nonoxidative glucose consumption during focal physiologic neuronal activity, Science 241(1988):462-4

[22] Gruetter, R., E. R. Seaquist, K. Ugurbil, A mathematical model of compartmentalized neurotransmitter metabolism in the human brain. Am. J. Physiol. Endocrinol. Metab. 281 (2001) E100-E112. 
[23] A. T. Gulledge, S. Dasari, K. Onoue, E. K. Stephens, J. M. Hasse, D. Avesar, A sodium-pump-mediated afterhyperpolarization in pyramidal neurons, The Journal of Neuroscience 33 (32) (2013) 13025-13041. doi:10.1523/ J. Neurosci.0220-13.2013.

[24] C. N. Hall, M. C. Klein-Flügge, C. Howarth, D. Attwell, Oxidative phosphorylation, not glycolysis, powers pre- and postsynaptic mechanisms underlying brain information processing, The Journal of neuroscience : the official journal of the Society for Neuroscience 32 (26) (2012) 8940-8951. doi:10.1523/J. Neurosci.002612.2012.

[25] L. Hertz,L. Peng, G. A. Dienel, Energy metabolism in astrocytes: high rate of oxidative metabolism and spatiotemporal dependence on glycolysis/glycogenolysis. Journal of Cerebral Blood Flow \& Metabolism, 27(2) (2007) 219-249.

[26] C. Howarth, P. Gleeson, D. Attwell, Updated energy budgets for neural computation in the neocortex and cerebellum, J.Cereb. Blood Flow Metab. 32 (7) (2012) 1222-1232. doi:10.1038/jcbfm.2012.35.

[27] G. Huguet, A. Joglekar, L.M. Messi, R. Buckalew, S. Wong, D. Terman Neuroprotective Role of Gap Junctions in a Neuron Astrocyte Network Model, Biophysical Journal, 11 (2) (2016), 452 - 462 doi:10.1016/j.bpj.2016.05.051.

[28] F. Hyder, R. K. Fulbright, R. G. Shulman, D. L. Rothman, Glutamatergic function in the resting awake human brain is supported by uniformly high oxidative energy, J. Cereb. Blood Flow Metab. 33 (3) (2013) 339-347. doi:10.1038/jcbfm.2012.207.

[29] Izhikevich EM. Dynamical Systems in Neuroscience: The Geometry of Excitability and Bursting (Computational Neuroscience) (2007) MIT Press.

[30] R. Jolivet, J.S. Coggan, I. Allaman, P.J. Magistretti. Multi-timescale modeling of activity-dependent metabolic coupling in the neuron-glia-vasculature ensemble. PLoS Comput Biol 11 (2) (2015) e1004036. doi:10.371/pcbi.1004036.

[31] J. Keener, J. Sneyd. Mathematical Physiology, Systems Physiology, (2009) second ed. Springer Verlag.

[32] B. King, A. P. Rizwan, H. Asmara, N. C. Heath, J. D. T. Engbers, S. Dykstra, T. M. Bartoletti, S. Hameed, G. W. Zamponi, R. W. Turner, Ikca channels are a critical determinant of the slow ahp in ca1 pyramidal neurons, Cell Reports 11 (2) 175-182. doi:10.1016/j.celrep.2015.03.026.

[33] A. Lajtha, Handbook of Neurochemistry and Molecular Neurobiology. Brain Energetics. Integration of Molecular and Cellular Processes, 3rd Edition, Springer US, 2007.

[34] C. Leithner, G. Royl, The oxygen paradox of neurovascular coupling, J.Cereb. Blood Flow Metab. 34 (1) (2014) 19-29. doi:10.1038/jcbfm.2013.181.

[35] I. Lundgaard, B. Li, L. Xie, H. Kang, S. Sanggaard, J.D.R. Haswell, T. Takano, R. Deane and M. Nedergaard. Direct neuronal glucose uptake heralds activity-dependent increases in cerebral metabolism. Nature Commun. 6 (2015) 6807.

[36] P. L. Madsen, R. Linde, S. G. Hasselbalch, O. B. Paulson, N. A. Lassen, Activation-induced resetting of cerebral oxygen and glucose uptake in the rat, J.Cereb. Blood Flow Metab. 18 (7) (1998) 742-748. arXiv:http://dx.doi.org/10.1097/00004647-199807000-00005, doi:10.1097/00004647-199807000-00005. URL http: //dx.doi.org/10.1097/00004647-199807000-00005

[37] F. A. Massucci, M. DiNuzzo, F. Giove, B. Maraviglia, I. P. Castillo, E. Marinari, A. D. Martino, Energy metabolism and glutamate-glutamine cycle in the brain: a stoichiometric modeling perspective, BMC Systems Biology 7 (1) (2013) 103. doi:10.1186/1752-0509-7-103. 
[38] M. Shah, D. G. Haylett, Ca2+ channels involved in the generation of the slow afterhyperpolarization in cultured rat hippocampal pyramidal neurons, J. of Neurophys. 83 (5) (2000) 2554.

[39] R. G. Shulman, F. Hyder, D. L. Rothman, Lactate efflux and the neuroenergetic basis of brain function, NMR in Biomedicine 14 (7-8) (2001) 389-396. doi:10.1002/nbm.741.

[40] R. G. Shulman, F. Hyder, D. L. Rothman, Insights from neuroenergetics into the interpretation of functional neuroimaging: an alternative empirical model for studying the brain's support of behavior, J.Cereb. Blood Flow Metab. 34 (11) (2014) 1721-1735. doi:10.1038/jcbfm.2014.145.

[41] E. Somersalo, Y. Cheng, D. Calvetti, The metabolism of neurons and astrocytes through mathematical models. Ann Biomed Eng 40 (2012) 2328-2344.

[42] E. Somersalo, R. Occhipinti, W.F. Boron and D. Calvetti, A reaction-diffusion model of $\mathrm{CO}_{2}$ influx into an oocyte. J. Theor. Biol. 309 (2012) 185-203.

[43] G.G. Somjen, H. Kager, W.J. Wadman. Computer simulations of neuron-glia interactions mediated by ion flux. J. Comput. Neurosci. 25 (2008), 349-365

[44] X.J. Wang, Calcium coding and adaptive temporal computation in cortical pyramidal neurons. J. Neurophysiol 79 (1998) 1549-1566. PMID: 9497431

[45] H. F. Wehrl, M. Hossain, K. Lankes, C.-C. Liu, I. Bezrukov, P. Martirosian, F. Schick, G. Reischl, B. J. Pichler, Simultaneous PET-MRI reveals brain function in activated and resting state on metabolic, hemodynamic and multiple temporal scales, Nat Med 19 (9) (2013) 1184-1189.

[46] F. W. Wehrli, Z. B. Rodgers, V. Jain, M. C. Langham, C. Li, D. J. Licht, J. Magland, Time-resolved mri oximetry for quantifying cmro(2) and vascular reactivity, Academic radiology 21 (2) (2014) 207-214. doi:10.1016/j.acra.2013.11.001.

[47] Wei Y, Ullah G, Schiff SJ. (2014) Unification of Neuronal Spikes, Seizure, and Spreading Depression. The Journal of Neuroscience. 34(35):11733-11743. doi: 10.1523/JNEUROSCI.0516-14/2014. 